Nouvelles perspectives en sciences sociales

\title{
Espace-temps d'un réseau sociocognitif complexe. II : Temporalités historiques et entropie sociocognitive
}

\section{Bernard Ancori}

Volume 4, numéro 1, septembre 2008

URI : https://id.erudit.org/iderudit/019639ar

DOI : https://doi.org/10.7202/019639ar

Aller au sommaire du numéro

Éditeur(s)

Prise de parole

ISSN

1712-8307 (imprimé)

1918-7475 (numérique)

Découvrir la revue

Citer cet article

Ancori, B. (2008). Espace-temps d'un réseau sociocognitif complexe. II : Temporalités historiques et entropie sociocognitive. Nouvelles perspectives en sciences sociales, 4(1), 9-76. https://doi.org/10.7202/019639ar

\section{Résumé de l'article}

L'espace d'un réseau sociocognitif complexe représenté dans un article précédent n'est qu'un épisode du temps de ce dernier dont les dimensions spatiales et temporelles sont ainsi indissociables. Sa dimension temporelle comporte plusieurs aspects liés. En effet, le temps subjectif de chaque acteur observé et le temps objectif de l'observateur du réseau nécessitent l'introduction d'un troisième terme pour pouvoir être pensés en tant que temps. Ce troisième terme est le temps comme tel, sous lequel sont subsumés temps subjectif et objectif. Le temps subjectif est ici celui d'acteurs internes au réseau et le temps objectif celui d'un observateur de ce dernier. Les rôles de l'acteur observé et celui de l'observateur du réseau étant interchangeables, ce texte suggère d'associer au temps comme telle la forme topologique d'une bande de Moebius. Plus généralement, le temps comme tel prend la forme d'une structure feuilletée d'une infinité de bandes de Moebius ordonnées selon une hiérarchie croissante de raccordements entre niveaux d'organisation d'un réseau qui peut en comporter un nombre infini. Ce texte analyse les modifications de cette structure lorsqu'elle est réduite à trois niveaux d'organisation. Dans le cadre d'une certaine continuité des temps subjectif et objectif au sein du réseau, ces modifications s'appliquent à une bande de Moebius donnée. Tel est le cas des périodes de « science normale » dans l'analyse kuhnienne de l'évolution scientifique, ou plus généralement dans tout processus d'apprentissage n'impliquant pas de bouleversement majeur. Mais lorsque cette continuité est rompue, par exemple à l'occasion d'une révolution scientifique, d'une conversion religieuse ou de tout autre rupture majeure dans un processus d'apprentissage, la bande de Moebius elle-même se trouve changée.
Ce document est protégé par la loi sur le droit d'auteur. L’utilisation des services d'Érudit (y compris la reproduction) est assujettie à sa politique d'utilisation que vous pouvez consulter en ligne.

https://apropos.erudit.org/fr/usagers/politique-dutilisation/ 


\section{Espace-temps d'un réseau sociocognitif complexe}

\section{II : Temporalités historiques et entropie sociocognitive}

Bernard Ancori

Université Louis Pasteur (Strasbourg 1)

Directeur de l'Institut de Recherches Interdisciplinaires

sur les Sciences et la Technologie (IRIST EA 3424)

Note du comité éditorial :

L'auteur poursuit ici la réflexion entreprise

dans le numéro précédent (volume 3, numéro 2).

Tel quil a été analysé dans le numéro précédent de cette 1 revue, l'espace d'un réseau sociocognitif complexe n'est jamais qu'un épisode du temps de ce dernier, de sorte que les dimensions spatiales et temporelles d'un tel réseau sont indissolublement liées. Comment représenter sa temporalité? La question de la représentation mathématique du temps historique n'est pas nouvelle, et chacun se souvient du couperet bergsonien à ce propos : "une équation algébrique exprime toujours un fait accompli. Or, il est de l'essence même de la durée et du mouvement, tels qu'ils apparaissent à notre conscience, d'être sans cesse en voie de formation ${ }^{1} »$. En matière de temporalité économique,

$\overline{1}$ Henri Bergson, Essai sur les données immédiates de la conscience, Paris, PUF, [1938] 1993, p. 89. 
cette question a reçu deux réponses très différentes. La première est celle du paradigme néoclassique, qui emprunte ses outils à la mécanique newtonienne (calcul différentiel et intégral) et se concentre le plus souvent sur une problématique de l'équili$b^{2}{ }^{2}$ : le temps n'est ici qu'une balise extrinsèque aux phénomènes dont il vient ponctuer la course, quand il ne se réduit pas purement et simplement à une caractéristique du bien économique 3 . À l'instar de la mécanique classique, le temps de l'économie néoclassique apparaît homogène, réversible et calculable, alors que le temps de l'histoire, irréversiblement scandé par des périodes hétérogènes, semble se jouer de tout calcul. Bref, cette représentation mathématique de la temporalité économique aboutit à vider cette dernière de tout contenu historique, et semble ainsi faire la preuve par l'absurde du bien-fondé de la position bergsonienne. La seconde réponse s'inspire de l'autre grande conception du temps présente dans la physique classique : celle de la deuxième loi de la thermodynamique (macroscopique) énonçant l'irréversibilité de la croissance de l'entropie de tout système isolé, et dont le fondement microscopique est fourni par la mécanique statistique ${ }^{4}$. Elle fut notamment explorée par Nicholas Georgescu-Roegen, qui transposa en économie les enseignements de l'expression macroscopique de l'entropie pour aboutir à une vision évolutionniste et dialectique du processus économique, mais non son interprétation statistique qu'il jugeait logiquement incohérente, du fait de l'impossibilité apparente d'expliquer des processus unidirectionnels par des lois statistiques indifférentes à la direction 5 .

$\overline{2}$ Giorgio Israël, La mathématisation du réel. Essai sur la modélisation mathématique, Paris, Seuil, 1996, p. 311-322.

3 Gérard Debreu, Théorie de la valeur. Analyse axiomatique de l'équilibre général, Paris, Dunod, 1966.

4 Pour une présentation récente de ces deux niveaux de lecture du concept d'entropie, ainsi que des liens existant entre eux, voir Roger Balian, «Le temps macroscopique ", dans Étienne Klein et Michel Spiro (dir.), Le temps et sa flèche, Paris, Flammarion, [1994] 1996, p. 155-211.

5 Nicholas Georgescu-Roegen, The Entropy Law and the Economic Process, Cambridge (Massachusetts), Harvard University Press, 1971, p. 159-169; Nicholas Georgescu-Roegen, La science économique. Ses problèmes et ses difficultés, Paris, Dunod, [1966] 1969, p. 76-84. 
De sorte que nous ne disposons toujours pas d'une modélisation unifiée transposant les interprétations micro et macroscopique de l'entropie dans le domaine de l'économique, et liant cette transposition à une analyse formalisée des temporalités historiques propres à ce domaine : " où est en économie l'équivalent de la deuxième loi de la thermodynamique, la tendance de l'entropie à croître (ou à rester la même) dans un système fermé? L'entropie est ce qui contribue à définir l'irréversibilité en physique, et nombreux sont les penseurs qui se sont essayés à définir une notion correspondante en économie, jusqu’à présent sans succès notable $e^{6}$ " Notre but est précisément de définir une telle notion, mais ceci en donnant au terme "économie " le sens explicité dans la première partie de ce texte : celui d'organisation des acteurs et de leurs interactions dans le cadre du réseau sociocognitif complexe qu'ils forment. C'est dans ce cadre précis que nous proposerons une analyse des temporalités historicoéconomiques et des significations que peuvent y revêtir les interprétations micro et macroscopique du concept d'entropie.

Nous procéderons ici en trois étapes. D’abord, nous suggérerons d'identifier le temps comme tel — sous lequel viennent se subsumer temps subjectif et objectif - à une forme topologique qui est celle de la bande de Moebius (II. 1). Ensuite, nous analyserons les formes de temporalité historique de notre réseau en liaison avec une interprétation de l'entropie sociocognitive propre à ce dernier (II. 2). Enfin, nous introduirons une représentation du type de rupture temporelle constitué par la réalisation d'un apprentissage batesonien de niveau trois, illustré ici par le cas particulier d'une révolution scientifique au sens de Thomas $S$. Kuhn (II. 3).

II. 1. Temps comme tel et bande de Moebius

Le concept de temps renvoie habituellement à deux dimensions considérées comme irréductibles : le temps subjectif de chacune de nos consciences, dont nous savons tous l'élasticité, tant est

$6 \quad$ Murray Gell-Mann, Le quark et le jaguar. Voyage au cour du simple et du complexe, Paris, Albin Michel, [éd. américaine : 1994] 1995, p. 360-361. 
parfois interminable le temps de l'attente et fugace celui du plaisir ; le temps objectif du jour et de la nuit, ou de nos montres. Apparemment irréductibles, ces deux dimensions sont pourtant inséparables, puisque l'élasticité de la première ne s'apprécie qu'à l'aune de la seconde et que la prétendue objectivité de cette dernière apparaît elle-même socialement construite ${ }^{7}$. À la fois irréductibles et inséparables, ces deux dimensions du temps nous apparaissent donc comme complémentaires. Nous rencontrons alors la position de Cornelius Castoriadis ${ }^{8}$ affirmant la nécessité d'un troisième terme pour pouvoir les penser simultanément : de même que la complémentarité de deux sous-ensembles ne s'apprécie que par rapport au référentiel offert par l'ensemble dont ils constituent une partition exhaustive, les dimensions subjective et objective du temps historique n'ont de sens que par rapport à un troisième terme. Ces dimensions réclament en effet la construction d'un concept de :

temps comme tel, $[. .$.$] troisième terme qui rend possible de parler \mathrm{du}$ temps subjectif et du temps objectif en tant que temps. Le temps comme tel apparaitrait ainsi comme surplombant non seulement les divers temps subjectifs [...] mais aussi tous les temps particuliers de quelque nature que ce soit, y compris le temps objectif et ses fragmentations possibles $[\ldots]$ et comme rendant possible, moyennant d'innombrables articulations et emboîtements croisés, leur ajustement réciproque, ou du moins leur accommodation, et leur correspondance ${ }^{9}$.

Comment concevoir et représenter ces trois facettes du concept de temps dans le cadre de notre modèle de réseau sociocognitif complexe? Commençons par la dimension subjective de ce concept - le " courant primitif de la conscience " dans lequel Georgescu-Roegen voyait la seule base du temps ${ }^{10}$ — en empruntant à Aristote sa fameuse formule selon laquelle « le temps est

$7 \quad$ Helga Nowotny, Le temps à soi. Genèse et structuration d'un sentiment du temps, Paris, Éditions de la Maison des sciences de l'Homme, [1989] 1992 ; Norbert Elias, Du temps, Paris, Fayard, [éd. allemande : 1984] 1996 ; Alain Adde, Sur la nature du temps, Paris, PUF, 1998.

8 Cornelius Castoriadis, Le monde morcelé. Les carrefours du labyrinthe III, Paris, Seuil, 1990.

$9 \quad$ Ibid., p. 247, italiques de l'auteur.

10 Nicholas Georgescu-Roegen, La science économique..., op. cit., p. 70 sq. 
le nombre du mouvement selon l'avant et l'après ${ }^{11}$ ". Bien qu'Aristote vienne d'affirmer que « le temps est le même, partout et en tous $»^{12}$, et semble avoir donné ainsi un caractère objectif à son concept, cette formule implique l'existence d'un être (d'une âme, psuché, dit Aristote) nombrant (mesurant) un changement (entendu en un sens très général) perçu par lui ${ }^{13}$ : dans un monde sans âme n'existerait nulle perception (et a fortiori nulle mesure) de changement, de sorte qu'il pourrait bien y avoir du mouvement en tant que "substrat " du temps, mais non du temps au sens plein. Comme le souligne en effet Castoriadis, "si rien ne change dans notre esprit, ou si le changement échappe à notre attention, il nous semble [...] que du temps ne s'est pas écoulél ${ }^{14}$ ». A contrario, dès lors que quelque chose change dans notre esprit et que ce changement attire notre attention, nous éprouvons la sensation d'un écoulement du temps. Dans notre modélisation, cela signifie que l'écoulement d'un temps subjectif est éprouvé par tout acteur dont le répertoire cognitif change, et qui mesure ce changement grâce à la capacité métareprésentationnnelle propre aux êtres humains ${ }^{15}$. Dans l'exemple donné dans la première partie de ce texte, il en va ainsi pour les acteurs $A_{k}$ et $A_{l}$, dont les répertoires cognitifs respectifs ont doublé de volume entre les dates $t_{0}$ et $t_{i}$.

Il est clair qu'une telle formulation implique immédiatement la dimension objective du temps historique, sans laquelle la notion de simultanéité des temps subjectifs, inhérente à l'expression de "dates $t_{0}$ et $t_{1}$ " communes à ces deux acteurs, serait vide de sens. Or, l'horloge que nous avons ainsi implicitement utilisée n'est nullement extrinsèque au réseau représenté. En effet, le temps objectif ainsi impliqué n'est autre que celui de l'observateur du réseau. Et ce dernier n'est pas (ne peut être) totalement extérieur au réseau lui-même : dans la théorie de la complexité

\footnotetext{
11 Physique, IV, 10, $219 \mathrm{~b}$ 1-2 ; 220a 24-25.

12 Physique, IV, 10, $218 \mathrm{~b}$.

13 Bernard Piettre, Philosophie et science du temps, Paris, PUF, 1994, p. 11-15.

14 Cornelius Castoriadis, Le monde morcelé..., op. cit., p. 257-258.

15 Dan Sperber (dir.), Metarepresentations: A Multidisciplinary Perspective, Oxford, Oxford University Press, 2000.
} 
naturelle, " cet observateur, extérieur au système, c'est en fait, dans un système hiérarchisé, le niveau d'organisation supérieur (englobant) par rapport aux systèmes éléments qui le constituent ${ }^{16}$. » Il suit qu'une durée objective s'écoule dans le réseau dès lors que du temps subjectif s'écoule pour un acteur observé au moins.

Nous entrevoyons ainsi une solution possible au problème de l'articulation de ces deux types de temps au temps comme tel, ce troisième terme indispensable pour que nous puissions parler des deux précédents en tant que temps. Notre rapide discussion sur la relation observateur/observé suggère en effet la possibilité d'une permutation entre ces deux niveaux hiérarchiques dans l'organisation de notre réseau complexe. À part leur différence de niveaux hiérarchiques, observateur et observé ne se distinguent en rien aux yeux du troisième homme que nous sommes - un observateur observant l'observateur observant le réseau. Observateur et observé se livrent en réalité au même type d'opérations cognitives (perception, observation, cognition interne, catégorisation, mémorisation, remémoration), et leurs positions sont parfaitement interchangeables, chaque acteur observé "à l'intérieur » du réseau étant simultanément un observateur situé à la limite de ce dernier, et réciproquement. La " représentation de représentations » en laquelle consiste notre modèle autorise donc sans difficulté la permutation de l'observateur et de l'observé.

Le caractère épistémologiquement périmé de la distinction ontologique classique entre sujet (observateur) et objet (observé)

16 Henri Atlan, Entre le cristal et la fumée. Essai sur l'organisation du vivant, Paris, Seuil, 1979, p. 70, italiques de l'auteur. Nous devons créditer cet observateur d'un niveau constant d'agraindissement pour pouvoir qualifier d'objectif le temps mesuré par lui, car l'horloge mesurant le temps objectif doit idéalement être elle-même (presque) intemporelle (Nicholas Georgescu-Roegen, La science économique..., op. cit., p. 73). C'est là tout à la fois une évidence théorique et une préoccupation pratique récurrente chez les physiciens, depuis Eddington disant que «meilleure est l'horloge, moins elle ressent le passage du temps » (Ibid.) jusqu’à Jean-Marc Lévy-Leblond affirmant que « toute réflexion sur la mesure du temps débouche [...] sur l'incontournable mais délicate dialectique du temps linéaire et du temps cyclique : il n'y a de temps que s'il y a changement, mais on ne peut le mesurer que s'il y a répétition sans changement» ("Quel temps fait-on? », dans Étienne Klein et Michel Spiro (dir.), Le temps et sa flèche, Paris, Flammarion, [1994] 1996, p. 277, italiques de l'auteur). 
se trouve ainsi souligné, alors même que cette distinction est réintroduite à un autre niveau : l'objet devient ici le couple observateur/observé, et le sujet le troisième homme évoqué plus haut. À la distinction ontologique classique entre sujet et objet se substitue ainsi une distinction opératoire entre deux niveaux hiérarchiques d'un seul et même objet — indispensable pour distinguer le temps subjectif (de l'acteur-objet observé) du temps objectif (de l'observateur objectivé). Corrélativement, le paradigme de la complexité naturelle réintroduit à un troisième niveau hiérarchique un sujet observant, et projette ainsi la dimension de l'énonciation (de l'observateur observé) dans l'espace de l'énoncé (du troisième homme). Aux yeux de ce dernier, la distinction entre temps subjectif (s'écoulant à l'intérieur du système) et temps objectif venant rendre simultanée (depuis l'« extérieur » du système) la pluralité d'événements qui s'y produisent, n'est donc pas exclusive d'un lien que ce troisième homme a pour tâche de tisser.

Ce lien est celui du temps comme tel, et l'une de ses figures possibles est celle d'une bande de Moebius. En effet, la topologie de cette dernière est caractérisée par le fait qu'elle ne possède qu'une seule face, au sens où il est possible de passer de son " intérieur " à son " extérieur" (et réciproquement) sans solution de continuité (sans passer par un bord). Or, c'est précisément de cette manière que peuvent permuter temps subjectif de l'observé "à l'intérieur" du réseau et temps objectif de l'observateur " extérieur " de ce réseau. Par ailleurs, on a pu montrer que la bouteille de Klein, cette forme topologique dont la structure est obtenue par raccordement de deux bandes de Moebius, figurait au plus près le type de spatialisation du temps qui se noue lors de l'intersubjectivité ${ }^{17}$. En définitive, le temps comme tel adopte donc la forme d'une bande de Moebius en liant les temps subjectifs des acteurs observés au temps objectif de l'observateur, alors qu'il prend celle d'une bouteille de Klein aux yeux du troisième homme en train d'observer l'intersubjectivité qui se

17 Eric Porge, Se compter trois. Le temps logique de Lacan, Ramonville, Éditions Érès, 1989. 
noue ainsi entre les deux niveaux d'organisation précédents — en train d'observer l'observateur observant. Et l'on peut imaginer ainsi une infinité potentielle de niveaux hiérarchisés d'observation - à un quatrième niveau se tiendrait un observateur observant l'observateur observant l'observateur observant les acteurs observés, et ainsi de suite — donnant au temps comme tel la structure feuilletée d'une infinité de bandes de Moebius ordonnées selon une hiérarchie croissante de raccordements.

Bien que sa mesure ait fait l'objet d'un long processus de standardisation en Occident, le temps historique n'a donc rien d'unique. Au contraire, il existe autant de temps subjectifs que d'acteurs et de temps objectifs que de niveaux d'observation dans l'organisation de la hiérarchie précédente : supposer le contraire serait postuler l'existence d'une sorte d'observateur en dernier ressort, cet imaginaire point de référence absolu auquel se situait Newton donnant le temps pour un continuum unidimensionnel dans lequel viendraient se ranger les événements selon un déterminisme causal universel et prédictible. La temporalité dont les trois facettes viennent d'être liées donne au contraire au temps comme tel un caractère historiquement construit, irréversible et incertain ${ }^{18}$.

Le temps est ici historiquement construit au sens où ce sont les modifications de répertoires cognitifs au sein du réseau qui font passer celui-ci d'une date quelconque à la date suivante, et non l'inverse. Et ce temps historique est irréversible au sens de Georgescu-Roegen ${ }^{19}$ qui distingue l'irréversibilité de l'irrévocabilité au sein de la classe des processus " non-réversibles " : à la différence d'un processus irréversible (telle la pousse et la tombée des feuilles d'un arbre), un processus irrévocable ne peut passer plus d'une fois par un état donné (telle la dégradation entropique de l'univers,

$\overline{18}$ L'incertitude ainsi évoquée est celle introduite par Frank H. Knight (Risk, uncertainty and profit, Boston, Houghton Mifflin Company, 1921) et John M. Keynes ("A Treatise on probability ", The Collected Writtings of. J. M. Keynes, vol. 8, Macmillan London for the Royal Economic Society, [éd. anglaise : 1921] 1973). Il s'agit donc d'une incertitude radicale, distincte du risque probabilisable. Cette caractéristique de la temporalité historique de notre réseau sera pleinement exploitée à la section 3 de cet article.

Nicholas Georgescu-Roegen, La science économique..., op. cit., p. 85-86. 
conçue par la thermodynamique classique). Or, le choix entre ces deux qualificatifs est manifestement lié au niveau d'agraindissement (au pouvoir de discrimination) qui est celui de l'observateur du processus en cause : plus ce niveau est élevé (plus ce pouvoir de discrimination est faible), plus c'est le terme d'irréversibilité qui doit prévaloir au détriment de celui d'irrévocabilité, et inversement. Imaginons en effet que ce niveau d'agraindissement soit nul, et que la carte devienne ainsi le territoire - pour reprendre la célèbre métaphore d'Alfred Korzybski. Il n'existerait alors aucune feuille semblable à aucune autre aux yeux de l'observateur doué de ce pouvoir de discrimination infini, et le processus de pousse et de tombée évoqué par Georgescu-Roegen serait parfaitement irrévocable, et non irréversible. Ce processus ne peut donc apparaître irréversible que dans la mesure où le niveau d'agraindissement de l'observateur est non nul : dès lors que son pouvoir de discrimination est fini, deux feuilles qui lui sembleraient différentes, s'il utilisait un niveau approprié d'agraindissement inférieur, lui paraissent au contraire suffisamment semblables pour qu'il décide de les ranger dans la même classe d'équivalence. En ce sens, le processus de la pousse et de la tombée concerne toujours la "même " feuille, et c'est pourquoi il apparaît irréversible.

La question qui surgit alors est celle de la conceptualisation et de la représentation analytique du phénomène de l'oubli dans les processus d'apprentissage. En effet, pour que le temps historique ici modélisé soit pleinement irréversible, l'oubli ne peut se réduire à un pur et simple effacement de parties figurant dans les répertoires cognitifs individuels. Car nous pourrions alors rencontrer une situation telle qu'un observateur qui ignorerait la trajectoire proprement historique du réseau (en n'observant que la suite discrète de ses états successifs) serait incapable de distinguer deux de ses états, dont l'un aurait pourtant précédé, et l'autre suivi, un état quelconque de celui-ci : il suffirait pour cela que les représentations nouvellement inscrites dans la mémoire globale du réseau soit exactement celles que viendrait effacer ensuite l'oubli. Cette apparente réversibilité (voire cyclicité) du 
temps objectif du réseau ne serait dissipée qu'en introduisant une échelle de temps calendaire totalement extérieure à ce dernier. La validité de notre concept de temps comme tel suppose donc d'exclure de notre représentation la possibilité de cette réversibilité, et nous devons ainsi donner à l'oubli un statut conceptuel qui aille au delà d'un effacement pur et simple d'information. Ce statut relève de la dimension intensive de l'apprentissage évoquée précédemment dans l'hypothèse H12. Nous savons en effet que l'apprentissage batesonien de niveau 2 peut déboucher sur une disparition de certaines catégories du niveau conscient des acteurs. Pareil enfouissement du trop connu dans les replis du non conscient peut être identifié ici à une fonction structurante de l'oubli dans tout processus d'apprentissage. En effet, l'oubli apparaît ainsi synonyme d'effacement et d'inscription simultanés d'information dans les répertoires des acteurs : l'effacement est celui d'une catégorie de type $C_{j} q$ au profit de l'inscription simultanée d'une catégorie $C_{j} q+1$, elle-même effacée ensuite au profit de l'inscription simultanée d'une catégorie $C_{j} q+2$, etc., jusqu'à atteindre une catégorie $C_{j} g-1$, dont l'effacement au profit de l'inscription simultanée d'une catégorie $C_{j} g$ signalerait que cette dernière rejoint le métaniveau sélectionnant et organisant au sein des répertoires cognitifs les sous-ensembles sémantiquement pertinents de ces derniers ${ }^{20}$. Nous développerons largement ce point dans la section II.

\section{2. Évolution du réseau et interprétations de l'entropie}

L'analogie entre le deuxième principe de la thermodynamique, tel qu'il fut exprimé initialement par Clausius au niveau macroscopique et la forme globale de l'évolution du réseau est relativement évidente. Ce principe stipule en effet que tout système isolé est le siège de transformations qui tendent irréversiblement à dégrader l'énergie libre qu'il contient (disponible pour fournir

$20 \quad$ Pour reprendre l'exemple avec deux acteurs présenté dans la première partie de ce texte, c'est ce métaniveau catégoriel qui, à la date $\mathrm{t}_{0}$, a déterminé les acteurs $A_{k}$ et $A_{1}$ à sélectionner respectivement les messages « $\mathrm{C}_{1} \mathrm{C}_{3}$ " et $" \mathrm{C}_{2} \mathrm{C}_{3}$ " parmi l'ensemble de messages potentiels contenant les huit parties figurant à cette même date dans leurs répertoires cognitifs respectifs. 
du travail) en énergie liée (en chaleur, qui ne peut plus être totalement retransformée en travail), et ceci jusqu'à atteindre un équilibre caractérisé par le fait que l'énergie libre y atteint sa valeur minimale : l'entropie d'un système isolé ne peut que croître et tendre ainsi vers un maximum qu'elle atteint lorsque le système est à l'équilibre thermodynamique. Néanmoins, l'activité physico-chimique productrice d'entropie fut ensuite envisagée d'un point de vue plus général ${ }^{21}$. C'est ainsi que l'état d'équilibre évoqué ci-dessus ne constitue aujourd'hui qu'un exemple particulier d'état stationnaire, c'est-à-dire d'état dont l'entropie ne varie pas au cours du temps ${ }^{22}$. Un tel état est obtenu lorsque la production d'entropie, qui mesure les processus irréversibles au sein du système envisagé par Clausius, est compensée en permanence par l'apport d'entropie, qui mesure les échanges de ce système avec son environnement. Alors que la production d'entropie est positive ou nulle, le signe de l'apport d'entropie dépend de la nature des échanges du système avec son environnement. Lorsque ces échanges sont tels que ce signe est négatif, production et apport d'entropie peuvent se compenser de telle sorte que le système se trouve dans un état stationnaire. Dans ces conditions, l'équilibre à la Clausius correspond au cas particulier où les échanges avec le milieu ne font pas varier l'entropie, et où la production d'entropie est donc elle aussi nulle.

Il en va formellement de même pour notre réseau cognitif. Dans la première partie de ce texte, nous l'avons considéré comme isolé, au sens où le seul moteur de changement y était la communication sociale parmi un nombre donné d'acteurs dont les répertoires cognitifs contenaient des listes données de catégories élémentaires. Le nombre de ces dernières ainsi que celui des acteurs étant finis, nous savons qu'il arrive inéluctablement un moment où cesse le jeu de recombinaisons en lequel consiste l'apprentissage de niveau 1 : celui où l'union des ensembles

$21 \quad$ Israël Prigogine et Isabelle Stengers, Entre le temps et l'éternité, Paris, Fayard, 1988, p. 49 sq.

22 Ce type d'état est également appelé « état stationnaire de non équilibre " pour bien marquer la permanence d'activités physico-chimiques en ce qui concerne le système considéré. 
représentant les répertoires cognitifs individuels coïncide avec l'ensemble des parties de l'ensemble des catégories élémentaires lui-même. Dans cet équilibre informationnel, il ne subsiste plus aucune différence entre les répertoires cognitifs des acteurs individuels, alors même que c'était la rencontre de telles différences lors de la communication sociale qui produisait à la fois l'extension des mémoires individuelles et l'accroissement du volume de la mémoire globale du réseau. En termes de l'exemple traité plus haut, cet équilibre informationnel était décrit par la matrice $\left[\mathrm{a}_{\mathrm{ij}}\right]\left(\mathrm{t}_{2}\right)$ suivante :

$\begin{array}{llllll} & \mathrm{C}_{1} & \mathrm{C}_{2} & \mathrm{C}^{3} & \mathrm{C}_{4} & \mathrm{C}_{5} \\ \mathrm{~A}_{\mathrm{k}} & 1 & 1 & 1 & 1 & 1 \\ \mathrm{~A}_{1} & 1 & 1 & 1 & 1 & 1\end{array}$

Le réseau était ainsi installé dans un équilibre informationnel où il dégénérait en une simple duplication d'un répertoire cognitif individuel. Lorsque ce type d'équilibre est atteint, toute différence cognitive interindividuelle est effacée, de sorte qu'aucun apprentissage extensif n'est désormais possible au sein du réseau, où ne subsistent donc que des possibilités d'apprentissages intensifs (de niveau 2 ou de niveau 3). Mais ces types d'apprentissages sont eux-mêmes bornés. Le caractère spéculaire de l'apprentissage de niveau 2 - tel celui qui transforme la catégorie $C_{3}^{2}$ en $C_{3}^{3}$ dans la période $\left(t_{1}, t_{2}\right)$ dans notre exemple - met en effet un terme à ce type d'apprentissage lorsque toutes les combinaisons de catégories élémentaires figurant dans la mémoire globale du réseau sont telles que $q=g$ pour tous les acteurs. La matrice des répertoires cognitifs individuels n'est alors plus observable, ni par suite le réseau lui-même, et non seulement nos acteurs sont devenus des clones parfaits, mais ils sont en outre totalement non conscients de cette triste condition : ce sont de purs automates répétant inlassablement le même message, identique pour tous car construit selon exactement les mêmes modalités par chacun, et ceci sans qu'ils le sachent.

Pour sa part, l'apprentissage de niveau 3 conduirait à modifier le mode de sélection des parties sémantiquement pertinentes 
$\mathrm{p}\left(S_{i}\right)(t)$ des répertoires cognitifs individuels $\mathrm{P}\left(S_{i}\right)(t)$, ainsi que les comportements d'émission et les modalités de réception des messages lors de la communication interindividuelle. Or, il ne peut pas non plus se réaliser, puisqu'en l'absence de processus de cognition interne ce type d'apprentissage ne peut résulter que de la confrontation de comportements individuels différents lors de la communication sociale, alors même qu'à l'équilibre informationnel du réseau tous ces comportements sont identiques. Ainsi dans l'exemple précédent nous avons supposé que ces comportements étaient invariants d'une période à l'autre : de $t_{1}$ à $t_{2}$, comme de $t_{0}$ à $t_{1}$, chaque acteur individuel émet un message consistant en une combinaison de deux catégories élémentaires exactement (dont l'une, déjà partagée, rend la communication possible, et l'autre, idiosyncrasique, est identiquement informative pour chaque partenaire de la communication). Cette invariance tient au fait que les comportements d'émission (mesurés par les volumes d'information émis) et de réception (évalués par les modes d'inscription de l'information reçue en mémoire) étaient strictement identiques pour les deux acteurs entre $t_{0}$ et $t_{1}$, et ils n'avaient donc aucune raison de changer entre $t_{1}$ et $t_{2}$. Il en va évidemment de même pour les périodes ultérieures, dont nous venons de voir qu'elles ne pouvaient connaître que des apprentissages de niveau 2 venant buter sur la limite correspondant à la disparition de la matrice aux yeux de l'observateur dans les conditions que nous avons décrites.

L'équilibre informationnel ainsi décrit correspond très exactement à l'équilibre thermodynamique tel que le concevait Clausius. Et il est facile de concevoir cet équilibre comme un cas particulier d'état stationnaire de notre réseau dès lors que nous considérons ce dernier comme ouvert - dès lors que la communication sociale n'y est plus le seul moteur de changement. En effet, comme nous l'avons vu précédemment, ouvrir notre réseau sociocognitif consiste à transformer les constantes $m$ et $n$ en variables, soit en introduisant des générations successives d'acteurs ( $\mathrm{m}$ devient variable), soit en ouvrant le réseau vers son intérieur (n varie du fait de l'existence de raffinements de caté- 
gories élémentaires existantes) ou vers son extérieur (n varie du fait de la création de nouvelles catégories élémentaires par les acteurs confrontés à l'environnement naturel du réseau). La production d'entropie du réseau nous apparaît donc résulter des processus irréversibles de communication sociale en son sein, et l'apport extérieur d'entropie provient des trois mécanismes évoqués à nouveau à l'instant. Ainsi que nous l'avions noté en concluant la partie précédente du texte, notre réseau sociocognitif peut alors se trouver, même avec un nombre constant d'acteurs donnés, dans un état stationnaire de non équilibre. Il suffit pour cela que le taux de socialisation des catégories idiosyncrasiques existantes, via la communication sociale au sein du réseau, soit égal au taux de création de nouvelles catégories idiosyncrasiques, par le raffinement de catégories existantes ou par l'introduction de nouvelles catégories idiosyncrasiques issues de la mise en acte d'analogies et de métaphores.

Dépassons à présent l'exemple simple rappelé ci-dessus pour réinterpréter l'évolution la plus probable de notre réseau cognitif représenté sous sa forme la plus générale en liaison avec l'entropie et les temporalités historiques correspondantes. Les développements précédents ont mis en évidence cinq caractéristiques centrales de cette évolution, lorsque son seul moteur est la communication sociale, que l'information est initialement répartie de manière équitable parmi les acteurs, et que tous ces derniers observent les mêmes comportements d'émission et de réception de l'information lors de leurs communications : 1) elle conduit à la formation d'amas différenciés de paires d'acteurs; 2) le caractère cumulatif de ce processus conduit chaque paire d'acteurs communicants vers un équilibre informationnel local; 3) toutes choses égales par ailleurs, lorsque deux acteurs (dont chacun est tiré d'un amas donné) auraient pu communiquer entre eux à la période précédente, mais ont au contraire communiqué avec l'acteur dont ils partagent à présent l'amas, la distance entre ces deux amas d'acteurs croît davantage que lorsque l'un de ces deux acteurs n'a communiqué avec personne; 4) les équilibres locaux vers lesquels tendent les amas d'acteurs ne sont pas irrévocables, 
contrairement à un équilibre informationnel global; 5) une ou plusieurs combinaisons de catégories élémentaires tendent à être partagées par l'ensemble des acteurs, instaurant ainsi progressivement un lien social dans le réseau considéré globalement, ce qui conduit ce dernier vers un équilibre informationnel global plutôt que vers une juxtaposition d'équilibres locaux dont chacun correspondrait à un amas composé de deux acteurs strictement identiques.

Quelles interprétations des temporalités historiques et de l'expression macroscopique de l'entropie pouvons-nous associer à une telle évolution? Du point de vue de la dimension subjective de la temporalité historique, nous pouvons immédiatement ajouter un énoncé à ceux produits auparavant. L'exemple de l'acteur $A_{m}$ lorsque $m$ est impair nous invite en effet, distinguer deux aspects dans cette dimension : d'une part, il semble n'exister aucun temps subjectif entre $t_{0}$ et $t_{1}$ pour cet acteur, dont l'exclusion momentanée de toute communication sociale laisse inchangé le répertoire cognitif individuel ; mais, d'autre part, nous avons vu les autres acteurs établir des communications dont le jeu combiné est venu déformer la structure des probabilités de communications ultérieures de l'acteur $A_{m}$ lui-même. De sorte que s'il n'existe pour $A_{m}$ aucun temps subjectif dans la dimension de l'être durant la période $\left(t_{0} t_{1}\right)$, il existe bel et bien pour lui un temps subjectif dans la dimension du devenir à la date $t_{1}$. De manière générale, il existe un temps subjectif dans la dimension du devenir de chaque acteur dès lors que ce type de temps existe dans la dimension de l'être d'au moins un acteur, c'est-à-dire dès que s'écoule une durée objective.

En dehors de cet énoncé directement lié à la tension dialectique inhérente à notre concept de propension à communiquer, nous pouvons reprendre ici, en les radicalisant, tous les énoncés produits précédemment. Ainsi, si les comportements individuels sont invariants lors des communications successives entre acteurs, le taux de production d'information ne peut que décroître au cours de l'évolution du réseau. En effet, le processus de formation d'amas évoqué à nouveau ci-dessus augmente la probabilité de 
communications de moins en moins informatives (car établies entre acteurs de plus en plus semblables au sein d'amas donnés) et diminue simultanément celle de communications potentiellement davantage informatives (car établies entre acteurs appartenant à des amas différents). Il suit que l'espérance mathématique du taux de production d'information via la communication sociale décroît de manière continue avec l'écoulement du temps objectif. Ce phénomène se poursuit jusqu'au moment où le jeu des recombinaisons cognitives produit par la communication vient se heurter à l'épuisement du réservoir de combinaisons virtuelles non encore actualisées. À ce moment, le réseau a atteint un équilibre informationnel global et le taux de production d'information devient nul.

Reprenons en effet la représentation générale de notre réseau sociocognitif à la date $t_{0}$ :

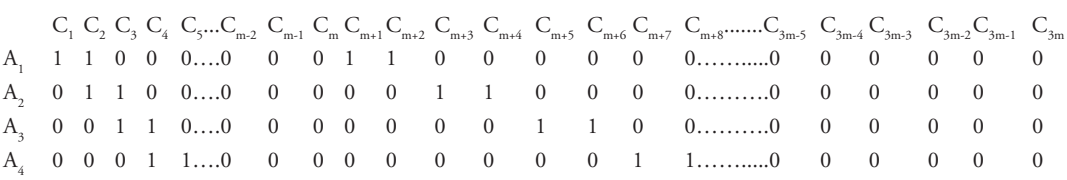

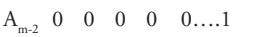

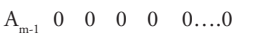

Dans cet état, chaque mémoire individuelle comporte 16 parties, et $H_{\text {max }}=16 \mathrm{~m}$ bits. Puisque les répertoires cognitifs de deux acteurs successifs contiennent une catégorie partagée, la complexité structurale du réseau est alors égale à $H=14 \mathrm{~m}$ bits. Il suit que la redondance $R$ du réseau est de 12,5\%. Avec $m$ impair, nous avions convenu plus haut que les couples entrant en communication entre $t_{0}$ et $t_{1}$ étaient les suivants : $\left(A_{1}\right.$ et $\left.A_{2}\right)$, $\left(A_{3}\right.$ et $\left.A_{4}\right),\left(A_{5}\right.$ et $\left.A_{6}\right), \ldots,\left(A_{m-2}\right.$ et $\left.A_{m-1}\right)$ - le $m^{\text {ième }}$ acteur étant provisoirement exclu de toute communication. Soient par exemple ( $" \mathrm{C}_{2} \mathrm{C}_{1}$ ") et $\left(" \mathrm{C}_{2} \mathrm{C}_{3}\right.$ ") les message émis respectivement $\operatorname{par} A_{1}$ et $A_{2}$ (et reçus respectivement par $A_{2}$ et $\operatorname{par} A_{1}$ ) lors de leur communication, et de même $\left("{ } \mathrm{C}_{4} \mathrm{C}_{3}\right.$ ") et $\left(" \mathrm{C}_{4} \mathrm{C}_{5}\right.$ ") pour ce qui concerne le couple $\left(A_{3}\right.$ et $\left.A_{4}\right)$, et ainsi de suite jusqu'à $\left(" \mathrm{C}_{\mathrm{m}-1} \mathrm{C}_{\mathrm{m}} »\right)$ et $\left(" \mathrm{C}_{\mathrm{m}-1} \mathrm{C}_{\mathrm{m}-2} »\right)$ pour le couple $\left(A_{m-2}\right.$ et $\left.A_{m-1}\right)$. 
À la date $t_{1}$, l'état du réseau est alors représenté par la matrice suivante :

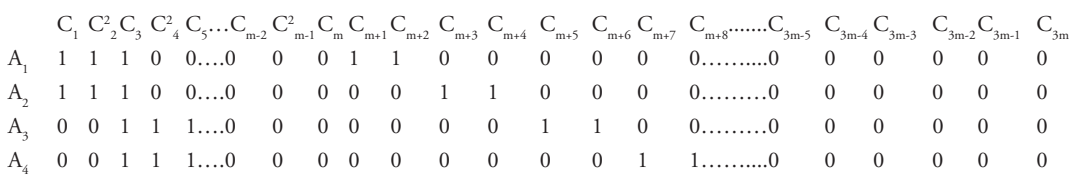

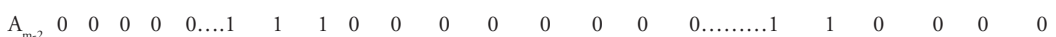

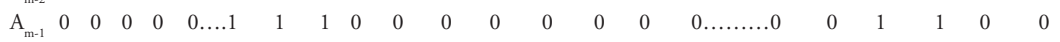
$\begin{array}{lllllllllllllllllllll}\mathrm{A}_{\mathrm{m}} & 1 & 0 & 0 & 0 & 0 \ldots .0 & 0 & 1 & 0 & 0 & 0 & 0 & 0 & 0 & 0 & 0 \ldots \ldots \ldots 0 & 0 & 0 & 0 & 1 & 1\end{array}$

Dans cet état, $H_{\max }=(32 \mathrm{~m}-16)$ bits, la complexité structurale du réseau $H=(27 \mathrm{~m}-12)$ bits, alors que $R=(5 \mathrm{~m}-4) /(32 \mathrm{~m}$ - 16). Par ailleurs, le taux de production d'information, mesuré par $\gamma\left(t_{0} t_{1}\right)=\left[H\left(t_{1}\right)-H\left(t_{0}\right)\right] / H\left(t_{0}\right)$, est égal à $[13(\mathrm{~m}-1)] /$ $(14 \mathrm{~m}+1)$. Avec $m=5, H_{\max }=144$ bits, $H=123$ bits, $R=14$, $58 \%$, et $\gamma\left(t_{0} t_{1}\right)=73,24 \%$.

Supposons à présent que les même couples d'acteurs entrent à nouveau en communication durant la période $\left(t_{1} t_{2}\right)$ - ce que nous savons être la structure de communications la plus probable - et que leurs comportements d'émission et de réception d'information soient alors identiques à ce qu'ils étaient précédemment - et nous savons qu'il n'y a aucune raison pour qu'il en aille autrement. Soient $\left(" \mathrm{C}_{2}^{2} \mathrm{C}_{\mathrm{m}+2}\right.$ ") et $\left(" \mathrm{C}_{2}{ }_{2} \mathrm{C}_{\mathrm{m}+3} »\right)$ les message émis respectivement par $A_{1}$ et $A_{2}$ (et reçus respectivement par $A_{2}$ et par $A_{1}$ ) lors de leur communication, et de même ( $" \mathrm{C}_{4}^{2} \mathrm{C}_{\mathrm{m}+6}$ ") et $\left(" \mathrm{C}_{4}^{2} \mathrm{C}_{\mathrm{m}+7} "\right)$ pour ce qui concerne le couple $\left(A_{3}\right.$ et $\left.A_{4}\right)$, et ainsi de suite jusqu'à (" $\mathrm{C}_{\mathrm{m}-1}^{2} \mathrm{C}_{3 \mathrm{~m}-4}$ ") et $\left(" \mathrm{C}_{\mathrm{m}-1}^{2} \mathrm{C}_{3 \mathrm{~m}-3}\right.$ ") pour le couple $\left(A_{m-2}\right.$ et $\left.A_{m-1}\right)$.

A la date $t_{2}$, l'état du réseau est alors représenté par la matrice suivante :

$\mathrm{C}_{1} \mathrm{C}_{2}^{3} \mathrm{C}_{3} \mathrm{C}_{4}^{3} \mathrm{C}_{5} \ldots \mathrm{C}_{\mathrm{m}-2} \mathrm{C}_{\mathrm{m}-1}^{3} \mathrm{C}_{\mathrm{m}} \mathrm{C}_{\mathrm{m}+1} \mathrm{C}_{\mathrm{m}+2} \mathrm{C}_{\mathrm{m}+3} \mathrm{C}_{\mathrm{m}+4} \mathrm{C}_{\mathrm{m}+5} \mathrm{C}_{\mathrm{m}+6} \mathrm{C}_{\mathrm{m}+7} \mathrm{C}_{\mathrm{m}+8} \ldots \ldots \mathrm{C}_{3 \mathrm{~m}-5} \mathrm{C}_{3 \mathrm{~m}-4} \mathrm{C}_{3 \mathrm{~m} \cdot \mathbf{3}} \mathrm{C}_{3 \mathrm{~m}-2} \mathrm{C}_{3 \mathrm{~m}-1} \mathrm{C}_{3 \mathrm{~m}}$ $\begin{array}{lllllllllllllllllllllll}\mathrm{A}_{1} & 1 & 1 & 1 & 0 & 0 \ldots .0 & 0 & 0 & 1 & 1 & 1 & 0 & 0 & 0 & 0 & 0 \ldots \ldots \ldots .0 & 0 & 0 & 0 & 0 & 0\end{array}$ $\begin{array}{lllllllllllllllllllllll}\mathrm{A}_{2} & 1 & 1 & 1 & 0 & 0 \ldots . & 0 & 0 & 0 & 1 & 1 & 1 & 0 & 0 & 0 & 0 \ldots \ldots \ldots 0 & 0 & 0 & 0 & 0 & 0\end{array}$ $\begin{array}{llllllllllllllllllllll}\mathrm{A}_{3} & 0 & 0 & 1 & 1 & 1 \ldots .0 & 0 & 0 & 0 & 0 & 0 & 0 & 1 & 1 & 1 & 0 \ldots \ldots \ldots 0 & 0 & 0 & 0 & 0 & 0\end{array}$ $\begin{array}{llllllllllllllllllllll}\mathrm{A}_{4} & 0 & 0 & 1 & 1 & 1 \ldots .0 & 0 & 0 & 0 & 0 & 0 & 0 & 0 & 1 & 1 & 1 \ldots \ldots \ldots . & 0 & 0 & 0 & 0 & 0\end{array}$ 
Dans cet état, $H_{\max }=(64 \mathrm{~m}-48)$ bits, $H=(47 \mathrm{~m}-32)$ bits, $R=(17 \mathrm{~m}-16) /(64 \mathrm{~m}-48)$, alors que $\gamma\left(t_{1} t_{2}\right)=(20 \mathrm{~m}-20) /$ $(27 \mathrm{~m}-12)$. Avec $m=5, H_{\text {max }}=272$ bits, $H=203$ bits, alors que $R=25 \%$ et $\gamma\left(t_{1} t_{2}\right)=65,04 \%$.

Enfin, supposons que les communications soient toujours du même type que précédemment durant la période $\left(t_{2} t_{3}\right): A_{1}$ communique " $\mathrm{C}_{2}^{3} \mathrm{C}_{\mathrm{m}+1}$ " à $A_{2}$ qui lui communique $" \mathrm{C}_{2}^{3} \mathrm{C}_{\mathrm{m}+4}$ ", $A_{3}$

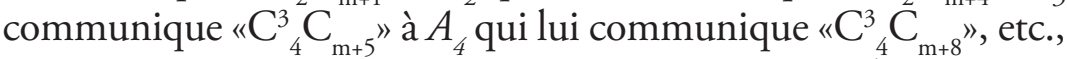
jusqu'à $A_{m-2}$ qui communique " $\mathrm{C}_{\mathrm{m}-1}^{3} \mathrm{C}_{3 \mathrm{~m}-5}$ " à $A_{m-1}$ qui lui communique $" \mathrm{C}^{3}{ }_{\mathrm{m}-1} \mathrm{C}_{\mathrm{m}-2}$ ".

À la date $t_{3}$, l'état du réseau est alors représenté par la matrice suivante :

\begin{tabular}{|c|c|c|c|c|c|c|c|c|c|c|c|c|c|c|c|c|c|c|c|c|}
\hline & $\mathrm{C}_{1}$ & $\mathrm{C}_{2}^{3}$ & & $\mathrm{C}_{4}^{3}$ & $\mathrm{C}_{5} \ldots . . \mathrm{C}_{\mathrm{m}}$ & $\mathrm{C}_{\mathrm{m}-1}^{3}$ & $\mathrm{C}_{\mathrm{m}}$ & $C_{m+1}$ & $\mathrm{C}_{\mathrm{m}+}$ & & $\mathrm{C}_{\mathrm{m}+4}$ & $\mathrm{C}_{\mathrm{m}+5}$ & & & $\mathrm{C}_{\mathrm{m}+8} \ldots \ldots . \mathrm{C}_{3 \mathrm{~m}-\mathrm{s}}$ & $C_{3 m-4}$ & $\mathrm{C}_{3 \mathrm{~m}-3}$ & $\mathrm{C}_{3 \mathrm{~m}-2}$ & $\mathrm{C}_{3 \mathrm{~m}}$ & $\mathrm{C}_{3 \mathrm{~m}}$ \\
\hline$A_{1}$ & 1 & 1 & 1 & 0 & $0 \ldots .0$ & 0 & 0 & 1 & 1 & 1 & 1 & 0 & 0 & 0 & $0 \ldots \ldots \ldots 0$ & 0 & 0 & 0 & 0 & 0 \\
\hline$\Lambda_{2}$ & 1 & 1 & 1 & 0 & $0 \ldots .0$ & 0 & 0 & 1 & 1 & 1 & 1 & 0 & 0 & 0 & $0 \ldots \ldots \ldots . .0$ & 0 & 0 & 0 & 0 & 0 \\
\hline $\mathrm{A}_{3}$ & 0 & 0 & 1 & 1 & $1 \ldots .0$ & 0 & 0 & 0 & 0 & 0 & 0 & 1 & 1 & 1 & $1 \ldots \ldots \ldots 0$ & 0 & 0 & 0 & 0 & 0 \\
\hline A & 0 & 0 & 1 & 1 & $1 \ldots 0$ & 0 & 0 & 0 & 0 & 0 & 0 & 1 & 1 & 1 & $1 \ldots \ldots \ldots . . .0$ & 0 & 0 & 0 & 0 & 0 \\
\hline
\end{tabular}

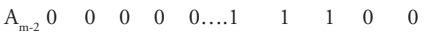

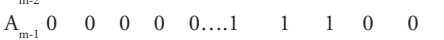

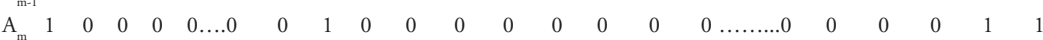

Dans cet état, $H_{\max }=(128 \mathrm{~m}-112)$ bits, $H=(63 \mathrm{~m}-48)$ bits, $R=(65 m-64) /(128 m-112)$, alors que $\gamma\left(t_{2} t_{3}\right)=(16 m-16)$ / (47m-32). Avec $m=5, H_{\max }=528$ bits, $H=267$ bits, alors que $R=42,99 \%$ et $\gamma\left(t_{2} t_{3}\right)=31,53 \%$.

Nous retrouvons ici la juxtaposition de $m-1$ équilibres informationnels locaux, analysée précédemment — où ils étaient atteints dès la date $t_{1}$ sous l'hypothèse d'acteurs mus par un souci de transparence totale entre $t_{0}$ et $t_{1}$. Entre l'état initial du réseau et ces équilibres informationnels locaux, atteints ici à la date $t_{3}$, le réseau est passé par quatre états séparés par trois périodes successives. Les tableaux 4 et 5 résument les valeurs ainsi obtenues par les principales caractéristiques du réseau pour un nombre $m$ d'acteurs égal à 5 . 
Tableau 4. Complexités structurales et redondances du réseau global pour $\mathrm{m}=5$

\begin{tabular}{|l|c|c|c|c|}
\hline Dates & $\mathbf{t}_{\mathbf{0}}$ & $\mathbf{t}_{\mathbf{1}}$ & $\mathbf{t}_{\mathbf{2}}$ & $\mathbf{t}_{\mathbf{3}}$ \\
\hline $\mathbf{H}_{\text {max }}$ (en bits) & 80 & 144 & 272 & 528 \\
\hline $\mathbf{H}$ (en bits) & 70 & 123 & 203 & 267 \\
\hline $\mathbf{R}$ (en \%) & 12,5 & 14,58 & 25 & 42,99 \\
\hline
\end{tabular}

Tableau 5. Valeurs du taux de production d'information du réseau global pour $\mathrm{m}=5$

\begin{tabular}{|c|c|c|c|}
\hline Périodes & de $t_{0}$ à $t_{1}$ & de $t_{1}$ à $t_{2}$ & de $t_{2}$ à $t_{3}$ \\
\hline$\gamma($ en $\%)$ & 73,24 & 65,04 & 31,53 \\
\hline
\end{tabular}

Ces tableaux montrent que la complexité structurale $H$ et la redondance $R$ du réseau ont augmenté de manière continue entre $t_{0}$ et $t_{3}$. Parallèlement, le taux de production d'information $\gamma$ n'a cessé de décroître. Comme nous l'avions remarqué précédemment avec notre exemple simple d'un réseau ne comptant que deux acteurs, la complexité structurale et la redondance du réseau varient dans le même sens tout au long de la trajectoire de ce dernier quand le seul moteur de son évolution consiste en une communication sociale dont tous les acteurs observent les mêmes comportements d'émission et de réception, ces comportements étant en outre invariants. En fait, $H$ mesure le volume de l'union des mémoires individuelles et $R$ mesure celui de leur intersection, et cette union et cette intersection croissent simultanément jusqu'à devenir parfaitement identiques à l'équilibre informationnel global du réseau ${ }^{23}$. Parallèlement, $\gamma$ ne cesse de décroître préci-

23 Ainsi que nous l'avons vu, aucun de ces équilibres informationnels locaux n'est irrévocable, du fait de leur destruction toujours possible par l'irruption de l'acteur $A_{m}$, resté jusque là isolé, dans le jeu de la communication. Entre la juxtaposition d'équilibres informationnels locaux, qui caractérise ici l'état du réseau à la date $t_{3}$, et l'équilibre informationnel global de ce dernier, prend place en effet le jeu de redéfinition des couples d'acteurs en communication vu précédemment. Parallèlement, un langage commun à l'ensemble des acteurs émerge progressivement, ce qui a pour effet de maintenir toujours ouverte la possibilité de communication entre acteurs appartenant à des amas 
sément du fait de la croissance continue de $R$ : tout se passe comme si le réseau voyait ses potentialités informatives étouffer de plus en plus sous le poids de sa redondance, et ceci jusqu'à ce qu'il atteigne un équilibre informationnel tel que $H_{\text {max }}=\mathrm{m} .2^{3 \mathrm{~m}}$ bits, $H=2^{3 \mathrm{~m}}$ bits, $R=1-(1 / \mathrm{m}) \%$ et où la valeur instantanée de $\gamma$ devient nulle. Avec $m=5: H_{\max }=\mathrm{m} .2^{15}, H=2^{15}, R=80 \%$, et $\gamma=0$.

En outre, les caractéristiques de cette évolution globale sont encore plus accusées au sein de chaque amas considéré isolément. Considérons en effet les deux tableaux suivants, qui donnent les valeurs des principales caractéristiques du réseau obtenues au sein de chacun des $m-1$ amas successifs de deux agents, pour $m=5$ :

Tableau 6. Complexités structurales et redondances de chaque amas pour $\mathrm{m}=5$

\begin{tabular}{|l|c|c|c|c|}
\hline Dates & $\mathbf{t}_{\mathbf{0}}$ & $\mathbf{t}_{\mathbf{1}}$ & $\mathbf{t}_{\mathbf{2}}$ & $\mathbf{t}_{\mathbf{3}}$ \\
\hline $\mathbf{H}_{\max }$ (en bits) & 32 & 64 & 128 & 256 \\
\hline H (en bits) & 30 & 56 & 96 & 128 \\
\hline $\mathbf{R}$ (en \%) & 6,25 & 12,5 & 25 & 50 \\
\hline
\end{tabular}

Tableau 7. Valeurs du taux de production d'information de chaque amas pour $\mathrm{m}=5$

\begin{tabular}{|c|c|c|c|}
\hline Périodes & ${\text { de } \mathbf{t}_{\mathbf{0}} \text { à } \mathbf{t}_{\mathbf{1}}}$ & de $\mathbf{t}_{\mathbf{1}}$ à $\mathbf{t}_{\mathbf{2}}$ & de $\mathbf{t}_{\mathbf{2}}$ à $\mathbf{t}_{\mathbf{3}}$ \\
\hline$\gamma(\mathbf{e n} \mathbf{\%})$ & 86,66 & 71,43 & 33,33 \\
\hline
\end{tabular}

Le tableau 6 ci-dessus montre que la redondance de chaque amas double de chaque date à la suivante. Avec une valeur initiale moitié moindre que celle du réseau global, elle rejoint cette dernière à la date $t_{2}$ pour la dépasser dès la date $t_{3}$. Et comme le

différents. Nous savons que ces deux phénomènes ont pour conséquence que l'état final du réseau est caractérisé par un seul amas d'acteurs tous strictement identiques, et correspond ainsi à un équilibre informationnel global irrévocable, et non par une juxtaposition d'amas composés chacun de deux acteurs strictement identiques, qui correspondraient à autant d'équilibres informationnels locaux. 
montre le tableau 7, c'est l'inverse qui se produit avec le taux de production d'information : s'il reste plus élevé que celui du réseau global durant nos trois périodes d'observation, de période en période le taux de production d'information de chaque amas décroît plus rapidement que celui du réseau global.

Qu'en est-il alors d'une interprétation possible de l'expression microscopique de l'entropie en ce qui concerne notre réseau sociocognitif complexe? Initialement exprimée par la formule de Boltzmann faisant de l'entropie une fonction du nombre de micro-états dans lesquels un système matériel dans un état macroscopique donné peut se trouver, l'approche microscopique de la loi d'entropie s'est ensuite enrichie de l'apport de Léon Brillouin ${ }^{24}$ introduisant explicitement, via l'information conçue comme néguentropie, le rôle de la mesure et de l'observateur dans la définition statistique de l'entropie. Davantage qu'une mesure de l'ordre microscopique du système considéré, cette dernière fut alors conçue comme une mesure du degré de connaissance qu'un observateur peut avoir, par des mesures expérimentales, de l'état microscopique de ce système. Plus précisément, en réécrivant la formule de Boltzmann en une formule de Shannon, il apparaît que l'entropie d'un système est proportionnelle à la quantité d'information qu'aurait l'observateur, s'il savait dans quel micro-état se trouve ce système, de sorte qu'elle mesure le manque d'information de l'observateur sur la véritable structure du système. Par suite, dire qu'un système isolé tend inéluctablement à évoluer vers l'état de désordre maximal est équivalent à dire que l'information dont dispose l'observateur de ce système ne peut que diminuer ${ }^{25}$.

L'évolution historique la plus probable de notre réseau mû par la seule communication sociale se prête remarquablement à une telle lecture. Le manque d'information de l'observateur sur la véritable structure de ce réseau est mesurable à la fois par la complexité structurale du système en cause, et par sa complexité

24 Léon Brillouin, La science et la théorie de l'information, Paris, Masson, 1959.

25 Henri Atlan, L'organisation biologique et la théorie de l'information, Paris, Hermann, 1972, p. 171-185. 
fonctionnelle. D'un point de vue de statique comparative, c'est bien sûr le concept de complexité structurale qui doit être utilisé, de sorte que l'entropie de chaque état du réseau est proportionnelle à la quantité d'information shannonienne qu'il contient dans cet état : dans la théorie des systèmes naturellement complexes, cette quantité mesure en effet l'information dont manquerait l'observateur pour reconstituer le système dans ses moindres détails s'il procédait au hasard ${ }^{26}$. En ce sens, l'évolution du réseau global témoigne d'un accroissement continu d'entropie entre son état initial et son état final, puisqu'à cette dernière date l'observateur aurait besoin de $m .2^{3 m}$ bits d'information pour reconstruire un état statistiquement identique à celui du réseau, alors qu'à la date initiale il n'aurait eu besoin que de $14 \mathrm{~m}$ bits pour le même type d'opération. Mais ce concept de complexité est manifestement trop pauvre pour nourrir une analyse véritablement dynamique de l'évolution de notre réseau en liaison avec l'expression microscopique de l'entropie, car il ne nous dit rien des relations fonctionnelles entre les noeuds de ce réseau. C'est ici d'un concept de complexité fonctionnelle que nous avons besoin.

Précisons la nature d'un tel concept dans notre analyse. Nous savons que chaque état réalisé du réseau est le résultat de l'application d'un ensemble de communications sociales dans l'état précédent, cet ensemble de communications sociales étant spécifié du point de vue de l'identité des acteurs en communication et de celui des valeurs informatives des messages alors échangés par eux. Nous pouvons donc mesurer la complexité fonctionnelle d'un état quelconque du réseau par la quantité d'information dont devrait disposer l'observateur de cet état pour être capable de prédire exactement l'état suivant parmi l'ensemble des états possibles. Or, nous savons que le taux de production d'information dans le réseau tend à diminuer avec le temps. Il s'ensuit qu'au fur et à mesure de l'allongement de la période d'observation, il manquera de moins en moins d'information à l'observateur du réseau afin d'être capable de prédire exactement l'état

26 Henri Atlan, Entre le cristal..., op. cit., p. 61-90. 
suivant de ce dernier parmi l'ensemble de ses états possibles. Et ceci jusqu'au moment final, où il ne lui en manque plus du tout : l'ensemble des états possibles se réduit alors à celui qui est réalisé, dont la prévision est par conséquent parfaite. Interprétée au niveau microscopique, l'entropie ne cesse donc de décroître dans le réseau, jusqu’à atteindre un niveau nul.

Comment concevoir les trois facettes de la temporalité historique du réseau en liaison avec cette interprétation de l'entropie? Nous savons que du temps subjectif s'écoule pour chaque acteur dont la ligne correspondante dans la matrice $\left[a_{i j}\right](t)$ se trouve modifiée. Une telle modification peut prendre deux formes : d'une part, celle d'une substitution de un ou plusieurs " 1 " à un ou plusieurs " 0 » (apprentissage extensif) ou inversement (effacement dû à l'oubli) dans la mémoire indivuelle concernée, et d'autre part, celle d'une modification du degré d'ancrage (mesuré par q) des catégories élémentaires présentes dans ces mêmes répertoires (apprentissage extensif). Or, la psychologie expérimentale a depuis longtemps mis en évidence l'existence d'une relation croissante entre la sensation d'écoulement du temps qu'éprouve le sujet individuel durant une période calendaire donnée et la richesse en événements qu'il perçoit durant cette même période ${ }^{27}$. Dans la dimension extensive de l'apprentissage, le taux de production d'information de l'acteur individuel par période calendaire constitue donc la variable qui détermine la vitesse d'écoulement du temps qu'il éprouve subjectivement durant cete période. Ce taux est mesurable par le taux de croissance du répertoire cognitif correspondant ${ }^{28}$. De ce point de vue, et sous

$27 \quad$ Sur cette relation psychologique entre sentiment de la durée et valeur événementielle des interactions du sujet avec son environnement, voir par exemple Lucien Colonna, "Le temps psychologique ", dans Adolphe Pacault et Christian Vidal (dir.), À chacun son temps, Paris, Flammarion, 1975, p. 214234.

28 Pour être complet, nous devrions également prendre en compte la possible décroissance de ce répertoire cognitif, inhérente à un effacement dû à l'oubli. Mais comment appréhender l'événement négatif que constitue ce dernier, puisque le sujet n'en a, par définition, pas conscience, de sorte qu'il échappe à l'ordre de ses métareprésentations? Nous traiterons donc ici cet événement négatif comme une absence d'événement, si insatisfaisante que soit une telle solution. 
notre hypothèse de comportements similaires et invariants de l'ensemble des acteurs durant toute la trajectoire du réseau, le temps subjectif s'écoule à une vitesse constante pour tous les acteurs, puisqu'il double de période en période pour chacun des acteurs des $m-1$ couples en communication, et que sa vitesse est uniformément nulle pour le $m^{\text {ième }}$ acteur qui reste isolé ${ }^{29}$.

Il n'en va pas de même pour le temps objectif — qui est le temps subjectif de l'observateur, qui observe l'ensemble du réseau, et non pas chaque acteur pris isolément. De son point de vue, puisque le taux de production d'information est continûment décroissant tout au long de la trajectoire du réseau, le temps s'écoule de plus en plus lentement. Ainsi, pour peu que nous dotions notre bande de Moebius d'une métrique appropriée, cela signifie que la taille de cette bande va sans cesse en diminuant : le nombre de changements observables dans la matrice $\left[a_{i j}\right](t)$ ne cessant de décroître d'une période à la suivante, tout se passe comme si le parcours nécessaire pour passer de l'intérieur à l'« extérieur» du réseau était de plus en plus court. Ce phénomène va en s'accentuant jusqu'à l'équilibre informationnel global du réseau, où la longueur de ce parcours devient nulle, de sorte que la bande de Moebius et la bouteille de Klein dégénèrent alors en un point. Observateurs et observés ne se distinguent plus alors en rien. Néanmoins, la productivité des raffinements de catégories élémentaires existantes et de la création de nouvelles catégories élémentaires peut être telle que la complexité fonctionnelle du réseau augmente, et son entropie également. L'observateur peut alors se consoler de la perte de prédictibilité correspondante en y voyant le signe même de l'inépuisable richesse d'une Histoire sans fin.

29 Bien entendu, les comportements d'émission des acteurs peuvent être différenciés au sein de chaque communication, du silence total (émission nulle d'information) à l'" aveu " complet (émission de messages comportant toutes les combinaisons présentes dans le répertoire cognitif considéré). La vitesse d'écoulement du temps subjectif de l'acteur récepteur individuel peut donc commettre des variations d'une période à l'autre. Nous pouvons cependant considérer que ces variations interindividuelles se compensent en moyenne, de sorte que le taux de production du réseau considéré globalement décroît de manière continue. 
II. 3. Apprentissage de niveau trois et rupture temporelle dans l'évolution du réseau : l'exemple d'une révolution scientifique à la Kuhn

Dans l'ensemble des développements précédents, nous avons analysé les différents aspects de l'évolution et de la temporalité historique du réseau dans le cadre d'un modèle de temps discret, sans en gommer pour autant toute notion de continuité. En effet, nous avons cantonné notre analyse de l'apprentissage aux niveaux 1 et 2 catégorisés par Gregory Bateson. Dans ce cadre, les acteurs individuels observent des comportements invariants en terme d'émission et de réception des messages lors de la communication, mais aussi à l'occasion de leurs processus de cognition internes. Ils interprètent notamment toujours de la même manière les événements dont ils enregistrent les significations dans leurs mémoires : ils lisent constamment l'autre dans le cadre du même. À l'inverse, lorsqu'ils effectuent un apprentissage batesonien de niveau 3, ces acteurs interprètent différemment des événements donnés, et lisent ainsi le même dans un cadre cognitif autre. Ce changement de comportement constitue une rupture temporelle radicale dans l'évolution du réseau, et c'est ce type de rupture que nous allons à présent analyser. Notre l'illustrerons par certains exemples issus de l'histoire des sciences, en soulignant d'emblée que la modélisation moderne des processus de révision des croyances n'établit guère de distinction entre la formation des croyances scientifiques et la formation d'opinions non scientifiques. En effet, ni les unes ni les autres :

n'ont $[\ldots]$ de fondement certain, mais résultent la plupart du temps d'un réarrangement de nos croyances précédentes face à de nouvelles informations. Nos tactiques rationnelles pour reconstruire en permanence nos croyances mêlent un souci d'accorder une priorité aux informations nouvelles et un souci de conservatisme et de mémoire dont le dosage dépend du contexte ${ }^{30}$.

Naturellement, les processus cognitifs et expérimentaux précis mis en œuvre par les scientifiques lors de la révision de leurs croyances different d'autant plus de ceux que nous activons

30 Denis Zwirn et Hervé Zwirn, "La révision des croyances ", Pour la science, $n^{\circ} 311$, septembre 2003, p. 69. 
quotidiennement dans la révision de nos opinions que le contexte évoqué par Denis Zwirn et Hervé Zwirn est analysé à un niveau fin. Mais au niveau de généralité qui est celui de notre analyse, nous pouvons confondre croyances scientifiques et opinions non scientifiques dans une théorie de la connaissance en général, conformément à la visée globale de ce texte. C'est dans cette perspective que nous allons présenter successivement trois processus distincts de révision des croyances que nous appellerons respectivement traduction, transformation faible et transformation radicale des croyances individuelles et collectives. Au prix d'un détour par la sémantique des mondes possibles, nous ferons ainsi mieux ressortir l'originalité du dernier de ces trois processus, qui constitue l'objet central de notre analyse.

\section{3.1. Traduction des croyances}

Nous définissons la traduction comme un processus de révision des croyances tel que l'ensemble des croyances anciennes est strictement contenu dans celui des croyances nouvelles. Appelons paradigme un ensemble de croyances partagées par un ensemble d'acteurs, l'emprise de ce paradigme pouvant être partielle (l'ensemble d'acteurs correspondant est un sous-ensemble de leur population globale) ou totale (l'ensemble de croyances considéré est partagé par toute la population d'acteurs). Illustrons ces définitions par une comparaison de l'état initial $\left(t_{0}\right)$ et l'état immédiatement suivant $\left(t_{1}\right)$ de notre réseau réduit à cinq acteurs. L'état initial $\mathrm{du}$ réseau, est alors représenté par la matrice suivante :

$\begin{array}{lllllllllllllllllll} & \mathrm{C}_{1} & \mathrm{C}_{2} & \mathrm{C}_{3} & \mathrm{C}_{4} & \mathrm{C}_{5} & & & & & & & & & & \mathrm{C}_{15} \\ \mathrm{~A}_{1} & 1 & 1 & 0 & 0 & 0 & 1 & 1 & 0 & 0 & 0 & 0 & 0 & 0 & 0 & 0 \\ \mathrm{~A}_{2} & 0 & 1 & 1 & 0 & 0 & 0 & 0 & 1 & 1 & 0 & 0 & 0 & 0 & 0 & 0 \\ \mathrm{~A}_{3} & 0 & 0 & 1 & 1 & 0 & 0 & 0 & 0 & 0 & 1 & 1 & 0 & 0 & 0 & 0 \\ \mathrm{~A}_{4} & 0 & 0 & 0 & 1 & 1 & 0 & 0 & 0 & 0 & 0 & 0 & 1 & 1 & 0 & 0 \\ \mathrm{~A}_{5} & 1 & 0 & 0 & 0 & 1 & 0 & 0 & 0 & 0 & 0 & 0 & 0 & 0 & 1 & 1\end{array}$

Dans cet état initial, nous pouvons identifier, par exemple, cinq paradigmes différents dont l'emprise partielle de chacun rassemble deux adhérents : $A_{1}$ et $A_{2}, A_{2}$ et $A_{3}, A_{3}$ et $A_{4}, A_{4}$ et $A_{5}$, 
$A_{5}$ et $A_{1}$. Examinons plus particulièrement les deux paradigmes auxquels adhèrent $A_{1}$ et $A_{2}$, d'une part, $A_{3}$ et $A_{4}$, d'autre part. Le premier (noté $\mathscr{C}_{1}$ ) est centré sur $C_{2}$, et ses interprétations par $A_{1}$ et $A_{2}$ sont formalisées par le reste des répertoires cognitifs respectifs de ces deux acteurs. Il en va de même pour le second (noté $\mathscr{C}_{2}$ ), centré sur $C_{4}$ et dont les interprétations sont formalisées par le reste des répertoires cognitifs des acteurs $A_{3}$ et $A_{4}$. Le socle cognitif de chacun de ces deux paradigmes consiste ainsi en la réunion des répertoires cognitifs de ses adhérents.

Moyennant la réalisation de certaines communications interindividuelles à la date $t_{0}$, l'état du réseau à la date $t_{1}$ est le suivant :

$\begin{array}{lllllllllllllllll} & \mathrm{C}_{1} & \mathrm{C}_{2}^{2} & \mathrm{C}_{3} & \mathrm{C}^{2}{ }_{4} & \mathrm{C}_{5} & & & & & & & & & & \mathrm{C}_{15} \\ \mathrm{~A}_{1} & 1 & 1 & 1 & 0 & 0 & 1 & 1 & 0 & 0 & 0 & 0 & 0 & 0 & 0 & 0 \\ \mathrm{~A}_{2} & 1 & 1 & 1 & 0 & 0 & 0 & 0 & 1 & 1 & 0 & 0 & 0 & 0 & 0 & 0 \\ \mathrm{~A}_{3} & 0 & 0 & 1 & 1 & 1 & 0 & 0 & 0 & 0 & 1 & 1 & 0 & 0 & 0 & 0 \\ \mathrm{~A}_{4} & 0 & 0 & 1 & 1 & 1 & 0 & 0 & 0 & 0 & 0 & 0 & 1 & 1 & 0 & 0 \\ \mathrm{~A}_{5} & 1 & 0 & 0 & 0 & 1 & 0 & 0 & 0 & 0 & 0 & 0 & 0 & 0 & 1 & 1\end{array}$

Chacun de nos deux paradigmes précédents s'est ainsi enrichi de croyances nouvelles, et il est clair que chaque nouvel ensemble de croyances ainsi formé contient strictement l'ancien ensemble de croyances correspondant. Les anciens paradigmes sont donc traduisibles en termes des nouveaux (notés (C)' 1 et (6)'2), conformément à notre définition de la traduction. La dynamique correspondante est celle d'un progrès scientifique par accumulation de connaissances. Cette dernière se traduit ici par le fait que ces nouvelles croyances sont formalisées par des répertoires cognitifs dont le volume global a doublé - et dont le noyau central partagé a plus que doublé tout en se voyant localement renforcé (les catégories $C_{2}$ et $C_{4}$ se sont vues respectivement remplacées par $C_{2}^{2}{ }_{2}$ et $C_{4}^{2}$ ) - par rapport aux répertoires cognitifs représentant les croyances initiales. Cette dynamique formalise ainsi la vision caractéristique de la philosophie des sciences "classique " (prékuhnienne) qui a dominé l'épistémologie jusqu'au " tournant historiciste " des années 1960. La vision carnapienne et la vision poppérienne se rejoignent en effet sur la notion de réduction 
d'une théorie à une autre : les concepts basiques de la première peuvent être définis en fonction des concepts basiques de la seconde, et les principes fondamentaux de celle-là sont déductibles logiquement des principes fondamentaux de celle-ci. Cette dernière est plus générale que la précédente, au sens où toutes les connaissances contenues dans la première sont aussi contenues dans la seconde ${ }^{31}$.

À l'instar de Robert Boyer et André Orléan ${ }^{32}$, nous retrouvons ainsi une notion d'accumulation dans la dynamique de la traduction. Mais à la différences du syncrétisme utilitariste affiché par ces deux auteurs, notre dynamique de la traduction se distingue nettement de la dynamique des " révolutions scientifiques " en se déroulant ici dans le cadre d'un paradigme donné, et non dans celui d'un changement de paradigme qui impliquerait une conception discontinuiste de l'histoire des sciences à la Kuhn ${ }^{33}$. En effet, dans l'exemple ci-dessus aucun des messages reçus par les quatre acteurs entre $t_{0}$ et $t_{1}$ n'est contradictoire avec les croyances initiales de ces acteurs, de sorte que ces messages s'intègrent à ces croyances par l'opération classique de conjonction (l'opérateur et) pour former de nouvelles croyances ${ }^{34}$.

31 Carlos Ulises Moulines, La philosophie des sciences. L'invention d'une discipline (fin XIXé-début XXI siècle), Paris, Éditions Rue d'Ulm/Presses de l'École normale supérieure, 2006.

32 Robert Boyer et André Orléan, " Persistance et changement des conventions. Deux modèles simples et quelques illustrations ", dans André Orléan (dir.), Analyse économique des conventions, Paris, PUF, 1994, p. 219-247.

33 Thomas S. Kuhn, La structure des révolutions scientifiques, Paris, Flammarion, [éd. américaine : 1962] 1972. Pour une conception davantage continuiste de l'histoire des sciences, voir par exemple Geoffrey Ernest Richard Lloyd, Les débuts de la science grecque de Thalès à Aristote, Paris, Maspéro, 1974; Magie, Raison et Expérience. Origines et développement de la science grecque, Paris, Flammarion, 1990; Pour en finir avec les mentalités, Paris, La Découverte, 1993.

34 La vision poppérienne de la réduction recèle une ambiguïté que la vision carnapienne ne contient pas : alors que Rudolph Carnap envisage surtout la comparaison entre théorie et observation en termes d'accroissement de la probabilité de la vérité de la théorie dans une perspective essentiellement vérificationniste (Logical Foundations of Probability, Chicago, University of Chicago Press, 1950), Karl Popper attend surtout que l'expérience ou la « discussion critique » viennent falsifier la théorie. Certes, conscient de certaines difficultés liées au falsificationnisme strict, le philosophe viennois introduit 
Ce type d'évolution est donc facilement formalisable dans le cadre de la logique classique appliquée à notre modèle. Cette logique est en revanche inapte à modéliser des modifications de croyances consécutives à la réception de messages contradictoires avec les croyances initiales. Afin de pouvoir traiter formellement ce type de situations, il faut quitter le cadre de la logique classique pour entrer dans celui de la sémantique des mondes possibles ${ }^{35}$. Ce cadre conceptuel a précisément été choisi il y a une vingtaine d'années par les logiciens pour formaliser quelques principes fondamentaux en matière de révision des croyances pouvant résulter de la réception de messages contradictoires avec les croyances initiales ${ }^{36}$.

\section{3.2. Révisions des croyances et sémantique des mondes possibles}

Suivons ici pas à pas Zwirn et Zwirn ${ }^{37}$ qui présentent les principes de la révision des croyances dans le cadre de la sémantique des mondes possibles ${ }^{38}$, et exprimons dans le langage de notre

le concept de « corroboration ", puis celui de " vraisemblabilité ", pour traiter le degré de confirmation apportée à une théorie ou à une hypothèse par l'observation (Objective Knowledge, Oxford, Clarendon Press, 1972), mais la perspective évolutionnaire réaffirmée dans ses derniers écrits (Toute vie est résolution de problèmes. Questions autour de la connaissance de la nature, Arles, Actes Sud, [éd. allemande : 1994] 1997) demeure centrée sur une vision falsificationniste de l'histoire des sciences. Or, $i$ ) le falsificationnisme implique l'existence et le constat de contradictions entre les croyances préalables des acteurs et l'information nouvelle qui leur est fournie par l'expérience ou la discussion critique; ii) le noyau logique de la réfutation du vérificationnisme par le falsificationnisme poppérien — l'erreur consistant à affirmer le conséquent dans un syllogisme Barbara - se situe dans le cadre strict de la logique classique : ce n'est que dans ce cadre strict que " ne pas être nécessairement vrai » est identique à " être faux ». D'où l'ambiguïté de la démarche poppérienne : l'opérateur de conjonction et de la logique classique ne permet pas de traiter formellement les situations dans lesquelles les croyances présentes des acteurs se trouvent confrontées à des informations nouvelles qui viennent les contredire.

35 Denis Zwirn et Hervé Swirn, «La révision des croyances », op. cit.

36 Jacques Dubucs, "La connaissance interactive ", Les chemins de la logique, Dossier Pour la Science, vol. 49, octobre-décembre 2005, p. 80-86.

37 Denis Zwirn et Hervé Swirn, "La révision des croyances ", op. cit.

38 Lensemble $W$ de tous les mondes possibles, ainsi que ses sous-ensembles, 
propre modélisation les différents processus ainsi impliqués. Parmi ces principes, deux sont particulièrement saillants : i) le message reçu - à l'occasion d'une communication sociale, ou de l'observation individuelle d'un objet - prime toujours sur la croyance initiale, et ce message n'est pas remis en cause à l'issue du processus de révision; ii) l'acteur rationnel suit un principe de changement minimal et cherche à préserver ses croyances les plus " enracinées $"^{39}$.

Le processus de révision dans lequel les croyances sont modifiées à propos d'un objet qui n'a pas changé, mais qui est à présent mieux connu, est appelé rectification par les logiciens ${ }^{40}$. La partie

peuvent être associés de façon biunivoque à des propositions, de sorte que raisonner dans le cadre des mondes possibles est équivalent à raisonner dans celui de la logique des propositions. Une croyance (ou un message) est ici l'ensemble des mondes possibles où cette croyance (ou ce message) est vraie. La conjonction de deux propositions est alors l'intersection des ensembles de mondes qui leur sont associés; la disjonction de deux propositions est l'union de tels ensembles, et la déduction de la proposition $p$ par la proposition $q$ signifie ici que l'ensemble des mondes associés à $q$ est inclus dans l'ensemble des mondes associés à $p$.

Introduite par Willard-V. Quine, la maxime de "mutilation minimale " exprime le fait que l'on «modifie ses croyances en présence d'une information contradictoire de façon à rétablir leur cohérence par le sacrifice des croyances auxquelles on est le moins attaché : Urbain Le Verrier a préservé ses croyances les plus "enracinées ", la théorie de Newton, en sacrifiant les moins "enracinées ", le nombre de planètes dans le Système solaire. Dans le processus de révision des croyances, il a suivi un principe de changement minimal. » (Denis Zwirn et Hervé Zwirn, op. cit., p. 66-67). Notons qu'une idée similaire se trouve au cœur de la conception comparative de la pertinence, introduite dans la théorie de la communication et de la cognition proposée par Dan Sperber et Deidre Wilson (La pertinence. Communication et cognition, Paris, Éditions de Minuit, [éd. anglaise : 1986] 1989). Selon cette conception, en effet, un énoncé est d'autant plus pertinent dans un contexte donné que l'effort nécessaire pour l'y traiter est moins important (Ibid., p. 181 sq.).

40 Carlos Alchourrón, Peter Gärdenfors et David Makinson ainsi que Denis Zwirn et Hervé Zwirn (op. cit.), présentent également les grandes lignes d'une autre modalité de révision des croyances, introduite par Hiroshi Katsuno et Alberto Mendelzon ( On the difference between updating a knowledge base and revisiting it », dans Peter Gärdenfors (dir.), Belief Revision, Cambridge, Cambridge University Press, 1992, p. 183-203), et appelée mise à jour des croyances. À la différence de la rectification, la mise à jour concerne des croyances qui évoluent face à un monde qui change. La plupart des axiomes 
axiomatique de la théorie de la rectification est fondée sur six propositions, dont la première énonce l'objectif de la théorie de la révision des croyances et les autres expriment, de différentes manières, la notion de changement minimal évoquée ci-dessus. Soient $K$ la croyance initiale, $A$ le message et $K^{*} A$ la croyance révisée. Les six axiomes sont alors les suivants : i) l'axiome de " succès » stipule que la cohérence des croyances doit être restaurée en cas de réception d'un message contradictoire avec les croyances initiales : $K^{*} A$ implique $A$, c'est-à-dire que la croyance révisée doit contenir le message ${ }^{41}$; ii) l'axiome de "conservation " dit que $K$ reste inchangé si $A$ se déduit de $K$; iii) l'axiome d'" inclusion " dit que $K^{*} A$ conserve la partie de $K$ compatible avec $A$; iv) l'axiome de " préservation » joint à l'axiome d' "inclusion " dit que si $K$ et $A$ sont compatibles, $K^{*} A$ est la conjonction des deux; v) l'axiome de "sub-expansion " et vi) l'axiome de "super-expansion " font intervenir deux messages successifs en étendant les intuitions de l'inclusion et de la préservation.

Ces deux derniers axiomes ne sont pas directement concernés par notre propos, qui est de modéliser les révisions de croyances consécutives à la réception d'un message ou à l'observation d'un objet à la fois - le réseau changeant corrélativement d'état. En revanche, il est facile de vérifier que notre modélisation de la

de la mise à jour sont identiques à ceux de la rectification, nonobstant un axiome supplémentaire (appelé " axiome de distributivité à gauche »), dont l'introduction est nécessaire car la mise à jour par un message non contradictoire avec les croyances initiales n'est plus une simple conjonction comme dans la rectification. Nous traiterons uniquement de cette dernière ici (et non de la mise à jour) car elle concerne un monde qui n'est pas supposé changer, tel le monde qui fait l'objet des sciences exactes. Pour une intéressante mise en relation de la théorie de la rectification avec la logique non monotone qui traite de raisonnements non certains, et pour une formalisation de l'abduction " ordonnée » issue des travaux de C. S. Peirce, voir (outre l'article utilisé ici) Bernard Walliser, Denis Zwirn et Hervé Zwirn, « Abductive logics in a belief revision framework ", Journal of Logic, Language and Information, vol. 14, $\mathrm{n}^{\circ} 1,2005$, p. 87-117.

41 Comme le notent ainsi Denis Zwirn et Hervé Zwirn (op. cit., p. 64, 66), Urbain Le Verrier, confronté aux observations d'Uranus contredisant les prévisions tirées de la théorie de Newton, obéit à l'axiome de succès lorsqu'il choisit de croire ces observations et postula l'existence d'une nouvelle planète pour rétablir la cohérence de la théorie. 
traduction vérifie les quatre premiers : i) les socles cognitifs des paradigmes $\sigma^{\prime}{ }_{1}$ et $\widetilde{C}^{\prime}{ }_{2}$ contiennent les messages échangés entre $t_{0}$ et $t_{1}$; ii) ces socles restent inchangés (au phénomène de renforcement près, sur lequel nous reviendrons) lorsque les messages reçus y sont déjà contenus; iii) ces socles conservent leurs parties compatibles avec les messages reçus — en fait, ils conservent ici toutes leurs parties, puisque les croyances initiales ne sont en rien contradictoires avec les messages reçus; iv) ces socles consistent en la conjonction des répertoires cognitifs initiaux et des messages reçus ${ }^{42}$.

Notre exemple simple d'un type particulier de révision des croyances au cours de l'évolution du réseau s'accorde donc parfaitement avec la partie axiomatique de la théorie de la rectification. Cela n'est guère étonnant puisque cet exemple est celui de cas où l'information nouvelle n'est pas contradictoire avec les croyances initiales, alors même que cette axiomatique a été élaborée pour rendre compte de processus de révision de croyance consécutifs à la réception de messages contradictoires ou non contradictoires avec les croyances initiales. Nous allons voir qu'il en va différemment avec la partie sémantique de la théorie de la rectification, dont certains aspects nous obligeront à dépasser le type particulier d'évolution examiné à travers l'exemple ci-dessus.

Cette partie sémantique repose sur la définition d'une relation de préférence, notée $<_{k}$, sur l'ensemble des mondes possibles : $w<_{k} w$ signifie que, étant donné $K$, le monde $w$ est plus plausible que le monde $w$ '. En d'autres termes, $w$ est sémantiquement plus proche que $w^{\prime}$ de la croyance initiale $K^{43}$. Cette hiérarchie

42 Denis Zwirn et Hervé Zwirn soulignent que cet énoncé plutôt trivial « montre que la théorie de la rectification, qui s'applique aux cas où le message contredit la croyance initiale, rejoint la logique classique lorsque l'information nouvelle n'est pas contradictoire. "(op. cit., p. 66). De fait, comme nous l'avons souligné plus haut, c'est bien parce qu'aucun des messages reçus par les quatre acteurs entre $t_{0}$ et $t_{1}$ n'est contradictoire avec les croyances initiales de ces acteurs que ces messages s'intègrent à ces croyances par l'opération classique de conjonction pour former de nouvelles croyances.

43 En revanche, face à n'importe quel monde extérieur à $K$, tous les mondes possibles de $K$ sont à égalité, et chacun d'eux est préféré au monde extérieur. 
de proximités sémantiques est représentée par un ensemble de couronnes emboîtées centrées sur $K$, de sorte que plus une couronne est proche de $K$, plus les mondes qu'elle contient sont plausibles compte-tenu de $K$. Le théorème de représentation établit alors « que l'opération logique de rectification satisfait les axiomes précédents si et seulement si la croyance révisée $K^{*} A$ est définie par l'ensemble des mondes de A qui coupe la couronne la plus proche de l'ensemble $K^{44}$ ».

La notion sémantique de proximité inhérente à la relation de préférence $<_{K}$ renvoie ainsi intuitivement aux propositions axiomatiques exprimant la notion de changement minimal, ce qui apparaît de manière plus claire encore dans l'énoncé de la suite du théorème : "lorsque le message $A$ contredit $K$, la croyance rectifiée $K^{*} A$ est l'intersection, disjointe de $K$, de $A$ avec la couronne la plus proche de $K$ que coupe l'ensemble des mondes de $A^{45}$ ». En d'autres termes, une proposition $A$ étant plus enracinée qu'une proposition $B$ si le premier monde possible qui contredit $B$ est plus proche de $K$ que le premier monde possible qui contredit $A$, la cohérence des croyances est rétablie conformément à la maxime de "mutilation minimale " de Quine : en sacrifiant les croyances les moins enracinées.

$44 \quad$ Denis Zwirn et Hervé Zwirn, op. cit., p. 66.

45 Ibid. 


\section{Figure $1^{46}$}

\section{AXIOMES DE LA RECTIFICATION}

orsque $K$ est la croyance, $A$ le message et $K^{\star} A$ la croyance révisée, la théorie de la rectification des croyances obéit notamment aux axiomes suivants:

L'axiome de succès:

$K^{*} A$ implique $A$.

L'axiome de conservation:

Si A se déduit de $K$, $K$ reste inchangé.

L'axiome d'inclusion:

$K^{\star} A$ conserve la partie de $K$ compatible avec $A$.

L'axiome de préservation joint à l'axiome d'inclusion:

$S i k$ et $A$ sont compatibles, $K^{\star} A$ est la conjonction des deux.

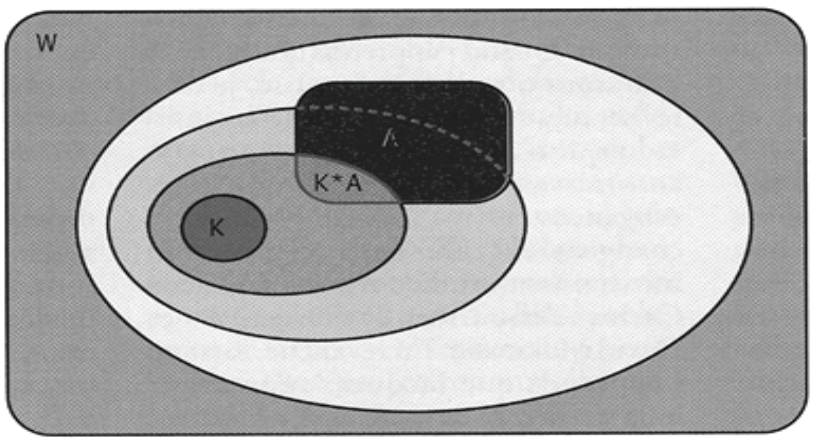

2. RA RECTIFICATION D'UNE CROYANCE $K$. Sur l'ensemble des mondes possibles $\mathrm{w}$ (en vert), on représente une relation de préférence entre différents ensembles de mondes possibles centrés sur la croyance $\mathrm{k}$ (le rond bleu représente l'ensemble des mondes possibles associés à $K$ ) par des couronnes emboîtées (en bleu): plus une couronne est proche de $\mathrm{K}$, plus les mondes qu'elle contient sont plausibles compte tenu de $\mathrm{K}$. En présence d'un message contradictoire A (en rouge), la croyance révisée est l'intersection de l'ensemble des mondes du message avec la couronne la plus proche de $K\left(K^{\star} A\right.$, en violet $)$. 
Figure $2^{47}$

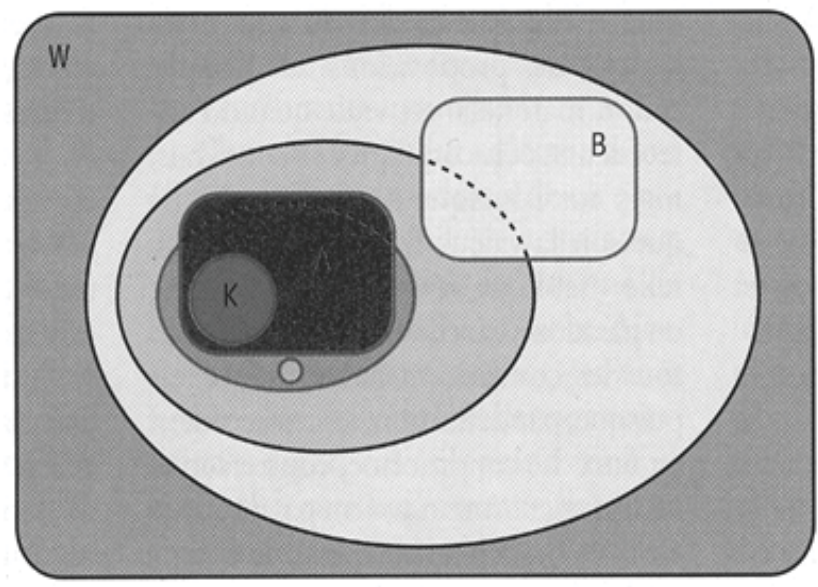

3. L'ENRACINEMENT D'UNE CROYANCE. L'ensemble des mondes possibles w (en vert) est doté d'une relation de préférence (les couronnes emboitées, en bleu) par rapport à une croyance $\mathrm{K}$ (le rond bleu). Dans cette relation, tout monde de $\mathrm{K}$ contredit la proposition B (en jaune), mais est en accord avec la proposition A (en rouge). Un des mondes qui contredit A est, par exemple, le point vert clair. Aussi, puisque le premier monde possible qui contredit $B$ (dans $k$ lui-même) est plus proche de $K$ que le premier monde possible qui contredit $\mathrm{A}$, la proposition $\mathrm{A}$ est plus enracinée que la proposition B.

Exprimons cette sémantique dans le langage de notre modèle. Dans la sémantique des mondes possibles, la notion de contradiction entre le message $A$ et la croyance initiale $K$ est formalisée par la disjonction des ensembles de mondes possibles de $K$ et des ensembles de mondes possibles de $A$. Dans notre modèle, cette contradiction se traduit par le fait que les catégories élémentaires figurant dans le message ne sont pas actuellement contenues dans l'ensemble des croyances. La réception de ce message provoque alors une recombinaison des catégories élémentaires qu'il contient avec les croyances initiales des acteurs récepteurs. En outre, dans l'hypothèse où la maxime de la mutilation minimale de Quine

$47 \quad$ Ibid., p. 93. 
est vérifiée, cette recombinaison est telle que les croyances les plus enracinées sont conservées au détriment de celles qui le sont moins. Dans notre modèle, la notion d'enracinement des croyances se traduit par le degré de renforcement des catégories élémentaires dans les répertoires cognitifs des acteurs, les différents degrés de renforcement étant formalisés par $0 \leq q<g$ dans les $C^{q}$ ainsi que nous l'avons vu précédemment. La maxime de Quine peut donc s'interpréter ici comme stipulant que les croyances initiales contenant des catégories élémentaires dont l'exposant est supérieur à l'exposant le plus élevé des catégories élémentaires contenues dans le message sont conservées au détriment d'autres croyances contenant des catégories élémentaires dont l'exposant est inférieur ou égal à cet exposant le plus élevé.

Par ailleurs, dans la théorie de la rectification, le processus selon lequel les acteurs renoncent à certaines croyances est appelé " contraction des croyances ". Un tel processus aboutit à restaurer la cohérence des croyances après l'éviction d'un message $A$ des croyances initiales $K$. Lidentité de Harper montre que les nouvelles croyances $K-A$, formées de l'union des croyances initiales $K$ et des croyances initiales rectifiées par la négation de $A$, consistent en l'intersection $K^{*}-A$ de $W-A$ avec la couronne la plus proche de $K^{48}$.

$48 \quad$ Ibid., p. 67. 
Figure $3^{49}$

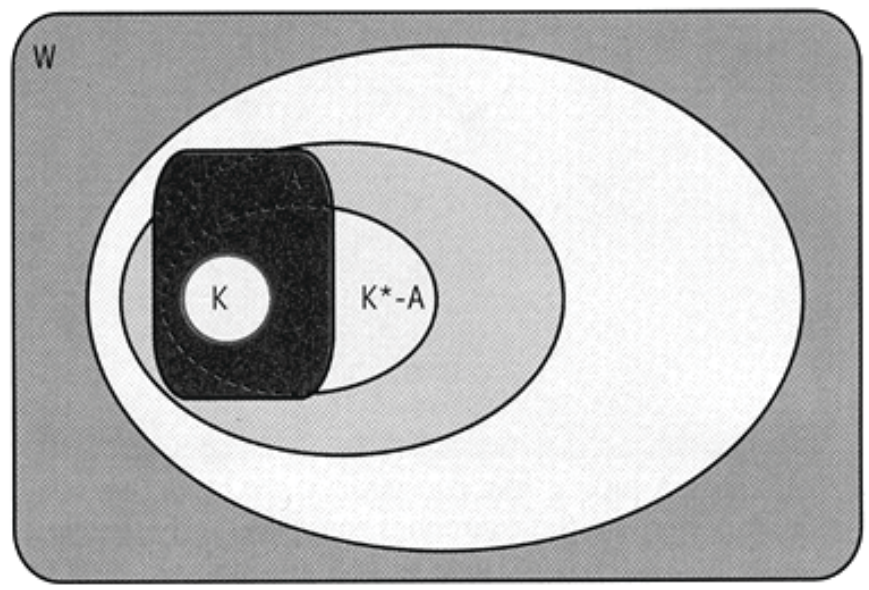

4. LA CONTRACIION D'UNE CROYANCE K (LE ROND) par un message A (en rouge) vise à rétablir la cohérence de $\mathrm{K}$ lorsqu'on renonce à $\mathrm{A}$. Selon I'identité de Harper, la nouvelle croyance K-A (en vert clair) est l'union de la croyance initiale et de la croyance initiale rectifiée par la négation de $\mathrm{A}$ (c'est-à-dire l'intersection de l'ensemble $\mathrm{W}$ dont on a enlevé A et de la couronne la plus proche de $\mathrm{K}$ ).

Dans notre modèle, ce sont certaines catégories élémentaires dont l'exposant est inférieur ou égal à l'exposant le plus élevé de celles contenues dans le message qui se voient ainsi évincées, et ceci d'autant plus que leur exposant est petit. En somme, notre formalisation traduit dans la troisième dimension de l'espace du réseau (qui est celle des valeurs de $q$ dans les $C^{q}$ ) la notion de proximité sémantique que la théorie de la rectification exprime par l'emboîtement hiérarchisé de couronnes centrées sur $K$ dans l'espace à deux dimensions qui est celui du plan. Il est donc possible d'exprimer dans le langage de notre modélisation les parties axiomatique et sémantique de la théorie de la rectification, et, dans ce cadre, les opérations cognitives impliquées par cette théorie s'effectuent clairement dans le cadre d'un paradigme donné lorsque le message reçu (ou le résultat de l'observation)

$49 \quad$ Ibid., p. 93. 
n'est pas contradictoire avec les croyances initiales.

Qu'en est-il lorsqu'il y a contradiction entre ces dernières et l'information nouvelle? Pour traiter ce point, nous devons distinguer désormais les répertoires cognitifs des acteurs envisagés d'un point de vue purement formel de la partie sémantiquement pertinente de ces répertoires dans chaque état du réseau. D’un point de vue formel, chaque répertoire cognitif est l'ensemble $\mathscr{P}\left(\mathrm{S}_{\mathrm{i}}\right)$ des parties de l'ensemble $S_{i}$ des catégories élémentaires connues de l'acteur $A_{i}$ dans l'état actuel du réseau. Mais d'un point de vue sémantique, seul un sous-ensemble $p \mathrm{~S}_{\mathrm{i}}$ de $\mathscr{P}\left(\mathrm{S}_{\mathrm{i}}\right)$ est pertinent dans cet état du réseau. Identifions désormais les croyances initiales de l'acteur $A_{i}$ à ce seul sous-ensemble $p \mathrm{~S}_{\mathrm{i}}$ dans chaque état donné du réseau. Lorsque l'information nouvelle est contradictoire avec les croyances initiales, nous assistons alors à un processus de recomposition des croyances qui aboutit à restaurer leur cohérence au prix d'un changement qualitatif : au sein de $p S_{i}$, certaines combinaisons de catégories élémentaires (dont le degré d'enracinement est supérieur à celui des catégories contenues dans le message ou l'observation véhiculant l'information nouvelle) sont conservées, d'autres (dont le degré d'enracinement est inférieur ou égal à celui des catégories contenues le message ou l'observation véhiculant l'information nouvelle) disparaissent et, finalement, la recombinaison des catégories élémentaires subsistantes avec celles que contient le message débouche sur une redéfinition de la partie sémantiquement pertinente des répertoires cognitifs.

Au sens strict, la traduction telle que nous l'avons définie ne consiste qu'en l'accumulation d'informations non contradictoires dans le cadre d'un paradigme donné. Dès que tel n'est pas le cas, le processus de redéfinition des croyances débouche ainsi sur de nouvelles croyances dont la cohérence suppose la disparition de certaines parties des croyances initiales. Le nouvel ensemble de croyances ne contient donc pas strictement l'ancien, dont il est qualitativement différent. Nous devons alors distinguer deux cas possibles : celui où cette différence qualitative s'exprime, comme c'était le cas de la traduction, dans le cadre d'un paradigme donné 
(au sens de $\mathrm{Kuhn}^{50}$ ), et celui où elle implique au contraire un changement de paradigme kuhnien. Dans le premier cas, nous parlerons de la transformation faible des croyances, et dans le second de transformation radicale. Dans le cas de la transformation faible, comme dans celui de la traduction, les processus concernés sont des apprentissages batesoniens de niveaux 1 et 2 réalisés dans le cadre de la science "normale»; dans celui de la transformation radicale, c'est à un apprentissage batesonien de niveau 3 que nous avons affaire, et il signale un changement de paradigme synonyme d'une révolution scientifique que Kuhn lui-même rapprochait d'une conversion religieuse.

II. 3.3. Transformation faible des croyances : l'apprentissage dans le cadre de la science normale

Prenons pour état initial des croyances celui qui prévaut à la date $t_{1}$ dans notre réseau sociocognitif :

$\begin{array}{lllllllllllllllll} & \mathrm{C}_{1} & \mathrm{C}_{2} & \mathrm{C}_{3} & \mathrm{C}^{2} & \mathrm{C}_{5} & & & & & & & & & & \mathrm{C}_{15} \\ \mathrm{~A}_{1} & 1 & 1 & 1 & 0 & 0 & 1 & 1 & 0 & 0 & 0 & 0 & 0 & 0 & 0 & 0 \\ \mathrm{~A}^{2} & 1 & 1 & 1 & 0 & 0 & 0 & 0 & 1 & 1 & 0 & 0 & 0 & 0 & 0 & 0 \\ \mathrm{~A}^{3} & 0 & 0 & 1 & 1 & 1 & 0 & 0 & 0 & 0 & 1 & 1 & 0 & 0 & 0 & 0 \\ \mathrm{~A}_{4} & 0 & 0 & 1 & 1 & 1 & 0 & 0 & 0 & 0 & 0 & 0 & 1 & 1 & 0 & 0 \\ A_{5} & 1 & 0 & 0 & 0 & 1 & 0 & 0 & 0 & 0 & 0 & 0 & 0 & 0 & 1 & 1\end{array}$

Restreignons-nous au paradigme $\mathscr{C}^{\prime}{ }_{1}$, auquel adhèrent $A_{1}$ et $A_{2}$, et dont le socle cognitif comporte, d'une part, la partie sémantiquement pertinente des représentations partagées par ces deux acteurs (formalisée par un sous-ensemble $p \mathrm{~S}_{\mathrm{C}}$ de $\mathscr{P} \mathrm{S}_{\mathrm{C}}$, ensemble des parties de l'ensemble $\left\{\mathrm{C}_{1}, \mathrm{C}^{2}{ }_{2}, \mathrm{C}_{3}\right\}$ ), et d'autre part, la partie sémantiquement pertinente des différentes interprétations de ce paradigme par les acteurs concernés (formalisées par un sous-ensemble $p S_{1}$ de $\mathscr{P} S_{1}-\mathscr{P} S_{\mathrm{C}}$ pour $A_{1}$, et par un sous-ensemble $p \mathrm{~S}_{2}$ de $\mathrm{S}_{2}-\mathscr{P} \mathrm{S}_{\mathrm{C}}$ pour $\left.A_{2}\right)^{l}$. Soient par exemple :

50 Thomas S. Kuhn, La structure des révolutions scientifiques, Paris, Flammarion, [éd. américaine : 1962] 1972. 


$$
\begin{gathered}
p \mathrm{~S}_{\mathrm{C}}\left(\mathrm{t}_{1}\right)=\left\{\mathrm{C}_{2}^{2}, \mathrm{C}_{3}, \mathrm{C}_{2}^{2} \mathrm{C}_{3}\right\} \\
p \mathrm{~S}_{1}\left(\mathrm{t}_{1}\right)=\left\{\mathrm{C}_{6}, \mathrm{C}_{2}^{2} \mathrm{C}_{6}, \mathrm{C}_{3} \mathrm{C}_{6}, \mathrm{C}_{2}{ }_{2} \mathrm{C}_{3} \mathrm{C}_{6}\right\} \\
p \mathrm{~S}_{2}\left(\mathrm{t}_{1}\right)=\left\{\mathrm{C}_{8}, \mathrm{C}_{2}^{2} \mathrm{C}_{8}, \mathrm{C}_{3} \mathrm{C}_{8}, \mathrm{C}_{2}{ }_{2} \mathrm{C}_{3} \mathrm{C}_{8}\right\}
\end{gathered}
$$

Supposons à présent que l'acteur $A_{1}$ crée une nouvelle catégorie élémentaire idiosyncrasique $C_{16}$ - par exemple, en formulant une analogie inédite ${ }^{51}$ - et communique (simultanément, pour plus de simplicité) le message " $\mathrm{C}_{2}^{2} \mathrm{C}_{16}$ " à l'acteur $A_{2}$. À la date $t_{2}$, le nouvel état du réseau est représenté par :

$\begin{array}{llllllllllllllllll} & \mathrm{C}_{1} & \mathrm{C}_{2}^{3} & \mathrm{C}_{3} & \mathrm{C}_{4}^{2} & \mathrm{C}_{5} & & & & & & & & \mathrm{C}_{15} & \mathrm{C}_{16} \\ \mathrm{~A}_{1} & 1 & 1 & 1 & 0 & 0 & 1 & 1 & 0 & 0 & 0 & 0 & 0 & 0 & 0 & 0 & 1 \\ \mathrm{~A}_{2} & 1 & 1 & 1 & 0 & 0 & 0 & 0 & 1 & 1 & 0 & 0 & 0 & 0 & 0 & 0 & 1 \\ \mathrm{~A}_{3} & 0 & 0 & 1 & 1 & 1 & 0 & 0 & 0 & 0 & 1 & 1 & 0 & 0 & 0 & 0 & 0 \\ \mathrm{~A}_{4} & 0 & 0 & 1 & 1 & 1 & 0 & 0 & 0 & 0 & 0 & 0 & 1 & 1 & 0 & 0 & 0 \\ \mathrm{~A}_{5} & 1 & 0 & 0 & 0 & 1 & 0 & 0 & 0 & 0 & 0 & 0 & 0 & 0 & 1 & 1 & 0\end{array}$

Supposons que le processus de rectification soit tel que :

$$
\begin{gathered}
p \mathrm{~S}_{\mathrm{C}}\left(\mathrm{t}_{2}\right)=\left\{\mathrm{C}_{2}^{3}, \mathrm{C}_{16}, \mathrm{C}_{2}^{3} \mathrm{C}_{16}\right\} \\
p \mathrm{~S}_{1}(\mathrm{t} 1)=\left\{\mathrm{C}_{6}, \mathrm{C}_{2}^{3} \mathrm{C}_{6}, \mathrm{C}_{6} \mathrm{C}_{16}, \mathrm{C}_{2}^{3} \mathrm{C}_{6} \mathrm{C}_{16}\right\} \\
p \mathrm{~S}_{2}\left(\mathrm{t}_{1}\right)=\left\{\mathrm{C}_{8}, \mathrm{C}_{2}^{3} \mathrm{C}_{8}, \mathrm{C}_{8} \mathrm{C}_{16}, \mathrm{C}_{2}^{3} \mathrm{C}_{8} \mathrm{C}_{16}\right\}
\end{gathered}
$$

La catégorie élémentaire $C^{2}$, davantage enracinée que la nouvelle catégorie $C_{16}$, subsiste sous la forme renforcée $C^{3}{ }_{2}$ dans le nouvel ensemble de croyances, alors que la catégorie $C_{3}$ a disparu de celui-ci, conformément à l'analyse ci-dessus. La restauration de la cohérence des croyances a nécessité la substitution de $C_{16}$ à $C_{3}$ dans toutes les combinaisons sémantiquement pertinentes au sein des répertoires cognitifs des acteurs. Il est clair qu'au sens strict, les nouvelles croyances ne contiennent pas les anciennes - bien que certains éléments de ces dernières aient subsisté, voire aient été renforcés, telle précisément $\mathrm{C}_{2}^{2}$ devenue $\mathrm{C}_{2}^{3}$ - mais constituent des ensembles qualitativement différents de ceux qui formalisaient ces dernières. La catégorie $C_{3}$ finira par disparaître plus ou moins rapidement des répertoires cognitifs

\footnotetext{
$51 \quad$ Bernard Ancori, Nouvelles perspectives en sciences sociales. Revue internationale d'analyse complexe et d'études relationnelles, vol. 1, $\mathrm{n}^{\circ} 1,2005$, p. 9-62.
} 
eux-mêmes - sauf à titre de vestige livré aux historiens, ou si la production d'une nouvelle catégorie lui redonne une pertinence sémantique par sa combinaison avec elle au sein des répertoires cognitifs des acteurs. Plutôt que de traduction du paradigme (ृ)' dans un paradigme $\mathscr{C}$ ", il vaut donc mieux parler de transformation du premier en le second pour désigner ce type de rectification des croyances. Et cette transformation peut être qualifiée de faible, car elle n'a pas le caractère de rupture radicale inhérent à un changement de paradigme à la Kuhn. En effet, rien ne dit que la substitution de $C_{16}$ à $C_{3}$ dans la partie sémantiquement pertinente des répertoires cognitifs des acteurs s'accompagne ici d'un changement dans le mode même de sélection et d'organisation des combinaisons de catégories élémentaires figurant dans les nouvelles croyances.

Afin de mieux le montrer, reprenons l'exemple d'Urbain Le Verrier utilisé par Zwirn et Zwirn ${ }^{52}$ pour illustrer leur présentation de la théorie de la rectification des croyances. Au XIX ${ }^{\mathrm{e}}$ siècle, les observations des mouvements d'Uranus (découverte en 1781 par Herschel) contredisent les prévisions tirées de la théorie de la gravitation élaborée à la fin du XVII ${ }^{e}$ siècle par Newton pour rendre compte des lois de Képler sur le mouvement des corps célestes. Plutôt que d'abandonner cette théorie, Urbain Le Verrier postule l'existence d'une nouvelle planète pour en rétablir la cohérence, et Neptune est découverte en 1846 par Johann Galle grâce aux prédictions de Le Verrier. Cette découverte " sauve " ainsi la théorie de Newton.

Identifions respectivement Urbain Le Verrier et Johann Galle à nos acteurs $A_{1}$ et $A_{2}$. Il est alors immédiat que $: i$ ) le noyau de la théorie de la gravitation de Newton est représentable ici par $C_{2}^{2}$; ii) dans l'hypothèse d'un Système solaire ne comportant que les six planètes connues de Newton (c'est-à-dire par ordre décroissant de proximité par rapport au Soleil : Mercure, Vénus, Terre, Mars, Jupiter et Saturne), cette théorie est représentable par $C_{2}^{2} C_{3}$; iii) cette combinaison de catégories élémentaires cesse d'être sémantiquement pertinente du fait des observations des

52 Denis Zwirn et Hervé Zwirn, "La révision des croyances ", op. cit. 
mouvements d'Uranus, de sorte que $C^{2}{ }_{2}$ ou $C_{3}$ doit être sacrifiée sur l'autel de la cohérence des croyances; iv) les calculs de Le Verrier prédisant l'existence d'une huitième planète ainsi que l'observation de Neptune par Galle sont représentables par $C_{16}$; v) la théorie de Newton, non seulement "sauvée ", mais aussi renforcée par la confirmation de l'existence de Neptune, est représentable par $C_{2}^{3} C_{16}$, nouvelle croyance venue se substituer à l'ancienne croyance $\mathrm{C}_{2}^{2} \mathrm{C}_{3}$ conformément à la maxime de Quine; vi) la catégorie $C_{3}$ n'est plus sémantiquement pertinente dans le registre de la physique théorique, bien qu' elle le demeure en glissant de ce registre à celui de l'histoire de la physique.

Ce processus de rectification des croyances s'inscrit dans le cadre strict d'un paradigme donné qui s'en trouve renforcé, et l'historien des sciences sait notamment que les conceptions newtoniennes de l'espace, du temps et du mouvement sont sorties intactes de ce processus. Dans le modèle proposé ici, la catégorie $C_{16}$ est certes venue se substituer à la catégorie $C_{3}$ dans la combinaison $C^{3}{ }_{2} C_{16}$ qui a succédé à $C_{2}^{2} C_{3}$ et représente désormais la théorie de la gravitation de Newton dans l'hypothèse où le Système solaire comporte huit planètes, et non plus sept (les six connues de Newton, plus Uranus et Neptune). Mais l'articulation interne de cette combinaison nouvelle n'a aucune raison d'être différente de celle de la combinaison qu'elle a évincée, car c'est bien dans le cadre même du paradigme newtonien que les calculs de Le Verrier, ainsi que l'observation ultérieure effectuée par Galle, ont prédit puis confirmé l'existence de Neptune. Dans les termes de notre modèle, ce qui précède signifie que les métacatégories non conscientes des acteurs n'ont été modifiées en rien lors du type particulier de rectification des croyances que nous venons d'analyser : quelle que soit le mode exact de la sélection et des articulations internes des parties sémantiquement pertinentes des répertoires cognitifs des acteurs, ce mode est resté identique à lui-même à l'issue de ce processus de révision des croyances. 
Pour l'instant, le modèle de la traduction de Boyer et Orléan ${ }^{53}$ recouvre donc indistinctement au moins deux processus que notre modélisation incite au contraire à considérer comme clairement différents. Le premier est celui de la traduction identifiée à une rectification des croyances consécutive à la réception d'une information nouvelle non contradictoire avec les croyances antérieures. Il se produit ici une accumulation de représentations lors de la révision des croyances des acteurs entre deux états successifs du réseau, et cette accumulation ouvre la possibilité d'une traduction du paradigme antérieur dans les termes du nouveau paradigme. À strictement parler, il n'y a cependant pas de transformation de ces représentations, mais uniquement une croissance purement quantitative des répertoires cognitifs concernés. L'intégralité de ces derniers peut ici être considérée comme étant sémantiquement pertinente, et identifiée aux croyances qui fondent les paradigmes successifs. En revanche, il s'avère nécessaire de distinguer les répertoires cognitifs des acteurs de leurs parties sémantiquement pertinentes dès lors que la rectification des croyances résulte de la réception d'une information nouvelle contradictoire avec les croyances antérieures. En effet, la restauration de la cohérence des croyances implique alors l'abandon de certaines croyances anciennes et leur substitution par certaines croyances nouvelles. En d'autres termes, certaines combinaisons de catégories élémentaires disparaissent immédiatement des nouvelles croyances, mais non nécessairement des répertoires cognitifs correspondants (rien ne s'oppose d'ailleurs à ce que ces combinaisons trouvent une autre pertinence sémantique dans le cadre d'autres paradigmes dont elles contribueraient ainsi à former les fondements cognitifs). Ce type de rectification implique donc une transformation qualitative des croyances sous-jacentes à une convention donnée, mais il ne peut être réduit à une accumulation puisqu'il comporte également une perte. Et comme le montre l'exemple que nous avons choisi pour l'illustrer, le résultat net de cette transformation faible des croyances, envisagée sous l'angle purement quantitatif de la comparaison des dimen-

53 Robert Boyer et André Orléan, "Persistance et changement... ", op. cit. 
sions respectives des parties sémantiquement pertinentes des répertoires cognitifs impliqués, peut très bien être rigoureusement nul - au renforcement de $C_{2}^{2}$ en $C^{3}{ }_{2}$ près.

\section{3.4. Transformation radicale d'un paradigme : l'apprentissage dans le cadre d'une révolution scientifique}

Il existe en outre une troisième catégorie de changement évoquée dans le modèle de la traduction de Boyer et Orléan ${ }^{54}$ : celui d'une "révolution scientifique " produisant un "nouveau paradigme " qui intègrerait «certains des traits et des propriétés de l'ancien paradigme, tout en lui étant supérieur ". Pour demeurer ici implicite, la référence à la vision kuhnienne de l'histoire des sciences n'en est pas moins claire. Malheureusement, ces deux auteurs entrent immédiatement ensuite en contradiction frontale avec les conceptions de Kuhn en affirmant l'existence d'une commensurabilité entre nouvelle et ancienne conventions ${ }^{55}$. En effet, logiquement cohérente avec la conception de la traduction d'une convention dans une autre proposée par Boyer et Orléan, cette notion de commensurabilité est absolument incompatible avec le concept de révolution scientifique introduit par un Kuhn passant les dernières années de sa vie à construire une théorie linguistique de l'incommensurabilité entre deux paradigmes séparés par une telle révolution ${ }^{56}$. Proposons alors une nouvelle dynamique possible des croyances, dont le fondement cognitif serait constitué par une rupture épistémologique analogue à celle qui sépare deux paradigmes kuhniens successifs. Et, de même que pour la rectification des croyances analysées précédemment, considérons qu'il n'y a ici guère de différence significative entre la psychologie du scientifique et celle du non scientifique en cette matière. Léna Soler souligne en effet que :

[d] ans la dernière phase de sa réflexion, Kuhn en vient à voir en l'incommensurabilité une conséquence de la nature même du fonctionnement systémique du

Ibid.

55 Ibid., p. 231.

56 Léna Soler, " Le concept kuhnien d'incommensurabilité reconsidéré à la lumière d'une théorie structurale de la signification ", Philosophia Scientiae, vol. $4, \mathrm{n}^{\circ}$ 2, 2000, p. 189-217. 
langage humain (l'incommensurabilité n'étant alors plus propre aux théories physiques ou même scientifiques, mais étant susceptible de s'appliquer entre deux systèmes théoriques quelconques portant sur le même objet) ${ }^{57}$.

Nous parlerons donc de transformation radicale pour désigner la dynamique séparant deux paradigmes successifs (scientifiques ou publics) dont les supports cognitifs respectifs sont incommensurables. Les exemples que nous choisirons pour illustrer une telle dynamique seront puisés dans l'histoire des sciences, mais au vu de ce qui précède nous pouvons raisonnablement supposer que les processus psychologiques impliqués dans une transformation radicale des paradigmes dépassent le cadre étroit des révolutions scientifiques pour s'appliquer à tout processus d'apprentissage batesonien de niveau $3^{58}$.

Introduit par Kuhn dès $1962^{59}$, le concept d'incommensurabilité renvoie tout d'abord à l'absence de commune mesure entre deux théories du même objet : ni les contenus de ces théories

57 Ibid., p. 193, italiques de l'auteur.

58 Une des principales différences entre paradigmes scientifiques et publics tient au fait que les controverses scientifiques débouchent plus généralement sur un consensus que les controverses publiques. Comme l'indique Thomas $S$. Kuhn dans la postface de 1969 à sa première édition, avant la transition de la période préparadigmatique à la période postparadigmatique, un certain nombre d'écoles se disputent la domination d'un secteur scientifique; après cette transition, ces écoles se réduisent en général à une seule, et s'instaure un mode de travail scientifique plus efficace - l'activité normale de résolution des énigmes devenant alors possible (op. cit., p. 211-212). Il reste que, même en matière scientifique, un tel processus peut être relativement long: durant toute la première moitié du XVIIIème siècle, il y eut presque autant de conceptions sur la nature de l'électricité que d'expérimentateurs importants dans ce domaine, et ce n'est qu'avec les travaux de Franklin et de ses successeurs immédiats que commença à se dégager un paradigme commun. Selon Kuhn, ce genre de situation est typique sur le plan historique : il en va de même pour l'étude du mouvement avant Aristote, de la statique avant Archimède, de la chaleur avant Black, de la chimie avant Boyle et Boerhaave, ou de la géologie historique avant Hutton (Ibid., p. 29 sq.).

59 Comme le rappelle Léna Soler ( $"$ The Incommensurability Problem: Evolution, Current Approaches and Recent Issues ", dans Léna Soler (dir.), Le problème de l'incommensurabilité, un demi siècle après, Éditions Kimé, 2004, p. 1-38), le concept d'incommensurabilité fut introduit simultanément et de manière indépendante par Thomas S. Kuhn et Paul Feyerabend (1962). Mais en ce qui concerne ce concept, l'histoire des sciences a davantage retenu la figure du premier que celle du second. 
(ontologies et significations respectives des êtres et des relations qui composent un paradigme) ni les normes de scientificité auxquelles elles répondent (objectifs, types de problèmes et de solutions, critères de la preuve, etc.) ne paraissent susceptibles d'être comparées dans le cadre d'un référentiel commun. Les nombreuses controverses portant sur ce concept qui semble menacer le réalisme scientifique, voire pencher vers le relativisme, incitent Kuhn, des années soixante jusqu’à sa mort, à le préciser et à le transformer. Il met notamment de plus en plus l'accent sur les différences de contenus théoriques (notamment, les changements de signification des termes qui interviennent dans les théories physiques) au détriment de celles qui portent sur les normes de scientificité.

Soler ${ }^{60}$ identifie sept éléments principaux constituant la dernière conception kuhnienne de l'incommensurabilité. Reprenons-en la liste, en explicitant la signification que chacun de ces éléments revêt dans le cadre de notre modélisation. Premièrement, la signification d'un signe, quel qu'il soit, a un caractère holistique, en ce qu'elle :

dépend avant tout des relations que ce signe entretient avec les autres signes du système linguistique où il intervient (plutôt que d'une connexion plus ou moins rigide à des états de choses extralinguistiques) ${ }^{61}$.

Ce caractère holistique apparaît dans notre modèle à travers la notion de pertinence sémantique des supports cognitifs d'une convention. Cette notion exprime en effet le fait qu'au sein de répertoires cognitifs donnés, seul un sous-ensemble de combinaisons de catégories élémentaires présente une signification pour les acteurs dans l'état considéré du réseau ${ }^{62}$. En d'autres termes,

\footnotetext{
$\overline{60}$ Léna Soler, « Le concept kuhnien d'incommensurabilité... », op. cit., p. 194196.

61 Ibid., p. 194.

62 Ainsi que l'a remarqué auparavant Léna Soler (Introduction à l'épistémologie, Paris, Ellipses, 2000, p. 193), Thomas S. Kuhn considère un paradigme scientifique " comme un ensemble d'énoncés plus ou moins étroitement connectés (en un sens très large du terme "énoncé" : phrases en mots, mais aussi expressions bien formées mettant en relation des symboles mathématiques) ». Expression bien formée mettant en relation des catégories élémen-
} 
la signification d'une catégorie élémentaire donnée dépend avant tout de ses relations avec d'autres catégories élémentaires au sein du support cognitif d'une convention, plutôt que de connexions "à des états de choses extra-linguistiques " ${ }^{63}$. Deuxièmement, ce caractère holistique reste néanmoins limité, car

déterminer la signification d'un terme n'oblige pas en pratique à prendre en compte la totalité du réseau linguistique auquel ce terme appartient : un fragment relativement restreint de ce réseau suffit en général ${ }^{64}$.

Il en va bien ainsi dans notre modèle où, par exemple, la détermination de la signification de $C^{2}{ }_{2}$ peut être restreinte à $p S_{c}$, fraction partagée par l'ensemble des acteurs des parties sémantiquement pertinentes de leurs répertoires cognitifs, sans référence aux divers $p \mathrm{~S}_{\mathrm{i}}$ formalisant les interprétations idiosyncrasiques de cette catégorie par ces mêmes acteurs. Troisièmement, les frontières d'un concept sont par essence indéterminées, car elles varient en fonction d'usages toujours fluctuants. Dans notre modèle, cette indétermination est précisément marquée, d'une part, par les différences entre les interprétations idiosyncrasiques de chaque catégorie élémentaire par les acteurs dans un état donné du réseau (indétermination synchronique) et, d'autre part, par les modifications de ces interprétations et du noyau partagé de significations impliquées par l'évolution du réseau (indétermination diachronique).

Quatrièmement, sur la base des trois premiers éléments ainsi recensés,

l'incommensurabilité est définie comme impossibilité de faire coïncider terme à terme les deux structures lexicales correspondant à deux para-

taires, la partie sémantiquement pertinente d'un répertoire cognitif entre donc dans la catégorie des énoncés ainsi entendus.

63 Même dans l'exemple de Le Verrier et Galle utilisé plus haut, c'est par la médiation d'une substitution de signes que le paradigme newtonien se voit faiblement transformé - en l'occurrence, par l'abandon de $C_{3}$ au profit de $C_{16}$ dans leurs combinaisons successives avec $C_{2}^{2}$ puis $C_{2}^{3}$. En effet, ce sont les calculs de Le Verrier qui conduisent à remettre en cause la pertinence sémantique de $C_{2}^{2} C_{3}$ et à postuler celle de $C_{2}^{3} C_{16}$, et l'observation ultérieure de Neptune par Galle ne devient opérante que par sa médiation dans le système linguistique des catégories, précisément sous la forme de $C^{3}{ }_{2} C_{16}$.

64 Léna Soler, "Le concept kuhnien d'incommensurabilité... ", op. ${ }^{16}{ }^{\circ}$ it., p. 194. 
digmes physiques ${ }^{65}$.

Comme le souligne immédiatement Soler, Kuhn parle ici d'impossibilité de traduire (italiques de l'auteur), ce qui conforte notre désaccord avec la position de Boyer et Orléan incluant les révolutions scientifiques dans leur modèle de la traduction. En effet, cette impossibilité signifie pour Kuhn

celle de remplacer un mot quelconque (ou une expression courte) d'un paradigme $P_{1}$ par un mot (ou une expression courte à peu près équivalente d'un paradigme $P_{2}^{66}$

Dans notre modèle de transformation faible d'un paradigme exposé précédemment, tel n'est évidemment pas le cas : dans l'exemple illustrant cette dynamique, la catégorie $C_{3}$ apparaît éminemment traduisible en $C_{16}$ dans le cadre d'un paradigme newtonien marginalement modifié par la substitution de $C^{3}{ }_{2} C_{16}$ à $C_{2}^{2} C_{3}$. C'est pourquoi nous avons pu regrouper la traduction (au sens strict que nous avons donné à ce terme) et la transformation faible au sein d'une même catégorie de processus de révision des croyances : celle qui repose sur des processus cognitifs relevant exclusivement des apprentissages batesoniens de niveaux 1 et 2 . L'apprentissage batesonien de niveau 3 sous-jacent à cet autre type de processus de révision des croyances que nous appelons ici transformation radicale (dont nous avons vu qu'une révolution scientifique à la Kuhn n'était qu'un exemple parmi bien d'autres) devra donc incorporer une forme d'impossibilité de traduire répondant à la définition de Kuhn revisitée par Soler.

Cinquièmement, cette incommensurabilité ne signifie pas qu'il est absolument impossible, pour un locuteur de $P_{2}$, de comprendre la signification et la raison d'être des affirmations de $P_{1}$ à propos du monde. Kuhn appelle interprétation le processus par lequel un locuteur de $P_{2}$ se déplace dans un système de pensée $P_{1}$ incommensurable au sien, et il identifie ce processus à l'apprentissage d'une langue étrangère. Soler ajoute à ce propos :

Dans le cas d'une langue étrangère, les mots rencontrés sont tout d'abord

65 Ibid.

66 Ibid. 
des inscriptions vides de sens. Dans le cas d'un paradigme physique incommensurable, les signifiants sont au contraire pour la plupart connus. Seulement, ils semblent employés d'une manière bizarre : les énoncés examinés et leurs enchaînements apparaissent tout d'abord étranges, voire incohérents. C'est que les mêmes termes n'ont en fait pas le même sens dans chacun des deux paradigmes incommensurables $P_{1}$ et $P_{2}^{67}$.

Dans le cadre de notre modèle, ce qui précède signifie que certaines catégories élémentaires sont conservées d'un ensemble de croyances au suivant, issu du précédent au terme d'une transformation radicale. D'autres catégories disparaissent au cours de cette dernière, et surtout, les nouveaux ensembles sémantiquement pertinents de combinaisons de catégories élémentaires ainsi formés ne sont plus sélectionnés ni organisés de la même manière que les anciens. Pour donner un exemple banal de ce type de transformation, les catégories de "temps " et $\mathrm{d}$ '" espace " présentes dans le paradigme newtonien $\left(P_{1}\right)$ sont conservées dans le paradigme einsteinien $\left(P_{2}\right)$, mais ceci sous forme d'une seule catégorie " espace-temps " insécable et relativement inédite, et non plus sous celle de catégories kantiennes absolues et bien distinctes que seule celle de mouvement permettait de lier depuis Galilée. Malgré la distance qui sépare ainsi la théorie gravitationnelle de Newton de celle d'Einstein, il est cependant possible, selon Popper,

de traduire la théorie newtonienne dans le langage d'Einstein, dans le formalisme de ce qu'on appelle le calcul tensoriel; et, si on le fait, à l'instar par exemple du professeur Peter Havas, on s'aperçoit que la différence entre les deux théories réside uniquement dans la vitesse de propagation finie de la gravitation, donc de la vitesse finie de la lumière c. Cela signifie que Havas a réussi à formuler la théorie d'Einstein, de telle sorte qu'en substituant une vitesse infinie à la vitesse de propagation finie $c$, elle se transforme en théorie newtonienne. Il serait cependant tout à fait illusoire d'en conclure que tout le progrès de la théorie d'Einstein réside dans la vitesse de propagation finie de la gravitation ${ }^{68}$.

La possibilité même d'une telle traduction tient à la relative

67 Léna Soler, "Le concept kuhnien d'incommensurabilité... ", op. cit., p. 195.

68 Karl Popper, Toute vie est résolution de problèmes, op. cit. 
proximité de l'univers culturel d'Einstein et de celui de Newton. Comme le souligne avec force Lévy-Leblond ${ }^{69}$, les différences entre Einstein et Galilée sont infiniment moins grandes que les différences entre Galilée et Aristote, et nous pouvons raisonnablement postuler que les différences entre Einstein et Newton sont encore moindres. Le fait que la traduction concrète de la théorie de l'un dans celle de l'autre soit alors partiellement possible résulte du caractère holistique limité de la signification du signe, qui fait de la langue un système quasi décomposable au sens de Herbert Simon ${ }^{70}$. Ce n'est que grâce à ce caractère limité et à ce caractère quasi décomposable que certaines parties de la théorie newtonienne sont traduisibles dans le langage einsteinien, car un holisme absolu de la signification du signe faisant de la langue un système strictement indécomposable aurait rendu toute traduction impossible. De manière générale, le bilinguisme (ainsi que Kuhn nomme la compétence acquise au terme du processus d'interprétation) n'implique nullement que la traduction soit possible. En réalité, elle l'est d'autant moins (même partiellement) que la distance entre les univers culturels dans lesquels s'enracinent les paradigmes successifs est grande. Ainsi,

[o]n peut $[\ldots]$ avoir réussi à interpréter la physique d'Aristote, être devenu capable de penser en aristotélicien, sans pour autant être en mesure de remplacer dans les écrits d'Aristote le terme de " mouvement " par un terme ou une expression du paradigme newtonien qui soit (même approximativement) équivalente ${ }^{71}$.

Sixièmement, l'incommensurabilité de deux paradigmes n’implique pas l'impossibilité de comparer leurs valeurs empiriques, notamment leurs efficacités prédictives mesurées par le nombre et la précision de leurs prédictions respectives. L'incommensurabilité

69 Jean-Marc Lévy-Leblond, "La structure de l'espace-temps (les relativités) ", dans Émile Noël (dir.), L'espace et le temps aujourd'hui, Paris, Seuil, 1983, p. $56-57$.

70 Herbert Simon, "The architecture of complexity ", Proceedings of the American Philosophy Society, vol. 106, 1962, p. 467-482. Reproduit dans Herbert Simon (dir.), Sciences des systèmes, Sciences de l'artificiel, Paris, Bordas, 1991, p. 171-203.

71 Léna Soler, "Le concept kuhnien d'incommensurabilité... ", op. cit., p. 195. 
kuhnienne ne débouche donc pas sur un relativisme.

Il faut au contraire affirmer l'existence d'un progrès scientifique, au sens où les hommes disposent effectivement d'outils toujours plus performants pour anticiper les événements naturels et agir sur le monde (et non pas au sens où les hommes accèdent à des descriptions toujours plus fidèles de la réalité indépendante) ${ }^{72}$.

En accord avec la position de Boyer et Orléan ${ }^{73}$ selon laquelle le nouveau paradigme intègre certains traits et propriétés de l'ancien, tout en lui étant supérieur, cette conception pragmatiste (et non, correspondantiste) de la vérité scientifique rejoint également l'un des fondements conceptuels de notre modèle, qui identifie les processus cognitifs à l'œuvre dans l'apprentissage batesonien à ceux d'un système adaptatif complexe au sens de Murray Gell-Mann ${ }^{74}$.

Septièmement, les adhérents d'un paradigme donné parviennent à communiquer grâce à la structure lexicale qui leur est commune, mais leurs critères d'identification des référents physiques des termes nommés ne sont pas nécessairement les mêmes. Peu visibles en période de science normale, ces différences le deviennent davantage au moment des révolutions scientifiques. Nous retrouvons ici la double face de chaque catégorie élémentaire dans les répertoires cognitifs des acteurs : sous forme de singleton, chaque catégorie élémentaire figure une forme minimale de langage commun permettant aux acteurs de communiquer ; idiosyncrasiquement combinée à d'autres, elle rend compte de la dépendance de la signification de l'information par rapport au contexte cognitif toujours singulier dans lequel cette information vient $s$ 'inscrire.

À la différence de Soler qui propose la théorie linguistique de Saussure pour penser l'incommensurabilité ainsi entendue ${ }^{75}$, la théorie du langage sous-jacente à notre modèle est plutôt d'inspiration peirceienne. Cela dit, notre objectif n'était pas ici de

Ibid., p. 196.

Robert Boyer et André Orléan, "Persistance et changement... ", op. cit. Murray Gell-Mann, op. cit.

Léna Soler, "Le concept kuhnien d'incommensurabilité... ", op. cit., p. 196 sq. 
discuter de la question de la meilleure théorie du langage en cette matière, bien que la voie ouverte par Charles $S$. Peirce présente l'avantage d'incorporer une dimension pragmatique absente de la linguistique structurale saussurienne. Plus modestement, il s'agissait plutôt pour nous de vérifier si notre modèle sociocognitif de la structure et du fonctionnement d'un réseau complexe incorporait bien les sept éléments principaux constituant, selon Soler, la dernière conception kuhnienne de l'incommensurabilité. Or, tel est bien est le cas, puisque ce modèle rend compte du caractère holistique d'un signe dont la signification ne requiert qu'une fraction de son réseau linguistique et qui, identifié à un concept dont les frontières sont indéterminées du fait d'usages fluctuants, débouche sur l'impossibilité de faire coïncider terme à terme les deux structures lexicales correspondant à deux paradigmes, sans barrer pour autant la possibilité d'une interprétation (mais interdisant généralement la traduction) d'un paradigme dans le cadre d'un autre - les valeurs empiriques de ces derniers pouvant être comparées, et leurs adhérents respectifs parvenant à communiquer, malgré leurs différences de critères d'identification des référents physiques des termes nommés.

Nous sommes donc en principe en mesure de développer à présent une analyse des processus sociocognitifs aboutissant à la transformation radicale d'un paradigme en un autre, incommensurable avec le précédent. Ces processus sont ceux qui gouvernent un apprentissage batesonien de niveau 3 débouchant sur une transformation du mode de sélection et d'organisation des combinaisons de catégories élémentaires sémantiquement pertinentes au sein des répertoires cognitifs des acteurs. De telles combinaisons constituant les fondements cognitifs successifs de paradigmes séparés par une transformation radicale, expliquer celle-ci revient à analyser le mode de succession de ceux-là. Donnons un exemple d'une telle transformation, en nous appuyant sur les principales phases du processus décrit par $\mathrm{Kuhn}^{76}$. Ce processus est grosso modo le suivant : i) la recherche

76 Thomas S. Kuhn, La structure des révolutions scientifiques, op. cit., p. 71135. 
dans le cadre d'un paradigme rencontre une anomalie, au sens où les résultats observés d'une expérience contredisent les résultats attendus dans le cadre du paradigme qui gouverne la science normale; ii) contrairement à ce qui se passe dans le cas de la transformation faible examiné plus haut, l'exploration de cette anomalie ne débouche pas sur un réajustement du paradigme tel que ce dernier reste lui-même, tout en incorporant désormais le phénomène anormal comme un phénomène attendu, mais sur le déclenchement d'une crise; iii) cette crise au sein du paradigme existant consiste précisément en l'incapacité plus ou moins prolongée dans laquelle se trouve ce dernier de se transformer faiblement; iv) après un certain temps, la crise est suivie d'une réponse prenant la forme d'une révolution scientifique; $v$ ) cette dernière est révélée par la formulation d'un nouveau paradigme, incommensurable avec l'ancien, et qui se montre apte à transformer le phénomène anormal en phénomène attendu.

Le paradigme initial peut, par exemple, être associé à l'état suivant de notre réseau sociocognitif à une certaine date $t$ :

$\begin{array}{lllllllllllllllll} & \mathrm{C}_{1} & \mathrm{C}_{2}^{2} & \mathrm{C}_{3} & \mathrm{C}^{2} & \mathrm{C}_{5} & & & & & & & & & \mathrm{C}_{15} \\ \mathrm{~A}_{1} & 1 & 1 & 1 & 1 & 0 & 1 & 1 & 0 & 0 & 0 & 0 & 0 & 0 & 0 & 0 \\ \mathrm{~A}_{2} & 1 & 1 & 1 & 1 & 0 & 0 & 0 & 1 & 1 & 0 & 0 & 0 & 0 & 0 & 0 \\ \mathrm{~A}_{3} & 0 & 1 & 1 & 1 & 1 & 0 & 0 & 0 & 0 & 1 & 1 & 0 & 0 & 0 & 0 \\ \mathrm{~A}_{4} & 0 & 1 & 1 & 1 & 1 & 0 & 0 & 0 & 0 & 0 & 0 & 1 & 1 & 0 & 0 \\ \mathrm{~A}_{5} & 1 & 1 & 1 & 1 & 1 & 0 & 0 & 0 & 0 & 0 & 0 & 0 & 0 & 1 & 1\end{array}$

Dans cet état du réseau, les cinq acteurs adhèrent au paradigme $\mathscr{C}_{1}$ dont le fondement cognitif comporte deux types de représentations : d'une part, la partie sémantiquement pertinente des représentations partagées par l'ensemble des acteurs, noyau dur du paradigme, formalisé par un sous-ensemble $p \mathrm{~S}_{\mathrm{C}}$ de $\mathscr{P} \mathrm{S}_{\mathrm{C}}$ qui est ensemble des parties de l'ensemble $\left\{\mathrm{C}_{2}^{2}, \mathrm{C}_{3}, \mathrm{C}_{4}{ }_{4}\right\}$; d'autre part, les parties sémantiquement pertinentes des interprétations idiosyncrasiques de ce paradigme par les acteurs, formalisées par un sous-ensemble $p \mathrm{~S}_{1}$ de $\mathscr{P} \mathrm{S}_{1}-\mathscr{P} \mathrm{S}_{\mathrm{C}}$ pour $A_{1}$, un sousensemble $p \mathrm{~S}_{2}$ de $\mathrm{S}_{2}-\mathscr{P} \mathrm{S}_{\mathrm{C}}$ pour $A_{2}$, etc. Soient, par exemple : 


$$
\begin{gathered}
p \mathrm{~S}_{\mathrm{C}}(\mathrm{t})=\left\{\mathrm{C}_{2}^{2}, \mathrm{C}_{3}, \mathrm{C}_{4}^{2}, \mathrm{C}_{2}^{2} \mathrm{C}_{3}, \mathrm{C}_{2}^{2} \mathrm{C}_{4}^{2}\right\} \\
\text { et } \\
p \mathrm{~S}_{1}(\mathrm{t})=\left\{\mathrm{C}_{6}, \mathrm{C}_{2}^{2} \mathrm{C}_{6}, \mathrm{C}_{3} \mathrm{C}_{6}, \mathrm{C}_{2}^{2} \mathrm{C}_{3} \mathrm{C}_{6}\right\} \\
p \mathrm{~S}_{2}(\mathrm{t})=\left\{\mathrm{C}_{8}, \mathrm{C}_{2}^{2} \mathrm{C}_{8}, \mathrm{C}_{3} \mathrm{C}_{8}, \mathrm{C}_{2}{ }_{2} \mathrm{C}_{3} \mathrm{C}_{8}\right\} \\
p \mathrm{~S}_{3}(\mathrm{t})=\left\{\mathrm{C}_{10}, \mathrm{C}_{2}^{2} \mathrm{C}_{10}, \mathrm{C}_{3} \mathrm{C}_{10}, \mathrm{C}_{2}^{2} \mathrm{C}_{3} \mathrm{C}_{10}\right\} \\
p \mathrm{~S}_{4}(\mathrm{t})=\left\{\mathrm{C}_{12}, \mathrm{C}_{2}^{2} \mathrm{C}_{12}, \mathrm{C}_{3} \mathrm{C}_{12}, \mathrm{C}_{2}^{2} \mathrm{C}_{3} \mathrm{C}_{12}\right\} \\
p \mathrm{~S}_{5}(\mathrm{t})=\left\{\mathrm{C}_{14}, \mathrm{C}_{2}^{2} \mathrm{C}_{14}, \mathrm{C}_{3} \mathrm{C}_{14}, \mathrm{C}_{2}^{2} \mathrm{C}_{3} \mathrm{C}_{14}\right\}
\end{gathered}
$$

Supposons à présent que l'acteur $A_{1}$ détecte une anomalie, au sens de Kuhn, et soit $C_{16}$ cette anomalie. Par définition, celle-ci n'est pas sémantiquement cohérente avec $p \mathrm{~S}_{\mathrm{C}}(\mathrm{t})$. Néanmoins, pour $A_{1}$ qui l'a détectée, elle serait sémantiquement cohérente, par exemple, avec $C_{7}$, qui figure bien dans son répertoire cognitif mais non dans $p \mathrm{~S}_{1}(\mathrm{t})$; en revanche, elle s'avère contradictoire avec $C_{6}$ qui, elle, figure dans $p \mathrm{~S}_{1}(\mathrm{t})$. à la date $t+1$, le nouvel état du réseau est alors représentable par :

$$
\begin{aligned}
& \begin{array}{llllll}
\mathrm{C}_{1} & \mathrm{C}_{2}^{2} & \mathrm{C}_{3} & \mathrm{C}_{4}^{2} & \mathrm{C}_{5}
\end{array} \\
& \begin{array}{lllllllllllllllll}
\mathrm{A}_{1} & 1 & 1 & 1 & 1 & 0 & 1 & 1 & 0 & 0 & 0 & 0 & 0 & 0 & 0 & 0 & 1
\end{array} \\
& \begin{array}{lllllllllllllllll}
\mathrm{A}_{2} & 1 & 1 & 1 & 1 & 0 & 0 & 0 & 1 & 1 & 0 & 0 & 0 & 0 & 0 & 0 & 0
\end{array} \\
& \begin{array}{lllllllllllllllll}
\mathrm{A}_{3} & 0 & 1 & 1 & 1 & 1 & 0 & 0 & 0 & 0 & 1 & 1 & 0 & 0 & 0 & 0 & 0
\end{array} \\
& \begin{array}{lllllllllllllllll}
\mathrm{A}_{4} & 0 & 1 & 1 & 1 & 1 & 0 & 0 & 0 & 0 & 0 & 0 & 1 & 1 & 0 & 0 & 0
\end{array} \\
& \begin{array}{lllllllllllllllll}
\mathrm{A}_{5} & 1 & 1 & 1 & 1 & 1 & 0 & 0 & 0 & 0 & 0 & 0 & 0 & 0 & 1 & 1 & 0
\end{array}
\end{aligned}
$$

À ce stade, l'anomalie peut raisonnablement être rejetée, car elle est isolée et n'a été détectée que par un seul acteur, qui la considère non significative et décide de l'ignorer. En ce cas, bien que son répertoire cognitif ait doublé de volume, la partie sémantiquement pertinente de ce répertoire ne change pas entre $t$ et $t+1$, et il en va naturellement de même pour les autres acteurs, dont les répertoires cognitifs n’ont pas été modifiés ${ }^{77}$. Mais il se peut également que cette anomalie soit si flagrante qu'elle attire

Soulignons qu'une telle stratégie cognitive est à l'opposé de celle que nous avons vu fonctionner dans le cadre de la rectification des croyances, où l'information nouvelle primait toujours sur les croyances antérieures. De fait, c'est la stratégie inverse qui fonctionne ici, et ceci selon une forme radicalisée de la maxime de Quine dans laquelle " mutilation minimale » équivaudrait à " absence totale de mutilation " en ce qui concerne des croyances confrontées à une information nouvelle qui vient les contredire. 
l'attention de l'ensemble des acteurs qui décident alors de l'examiner sérieusement. Toutes choses égales, par ailleurs, supposons ainsi que l'existence de l'anomalie $C_{16}$ soit progressivement confirmée par tous les acteurs ${ }^{78}$. À une certaine date $t+T$, le réseau est donc représentable par :

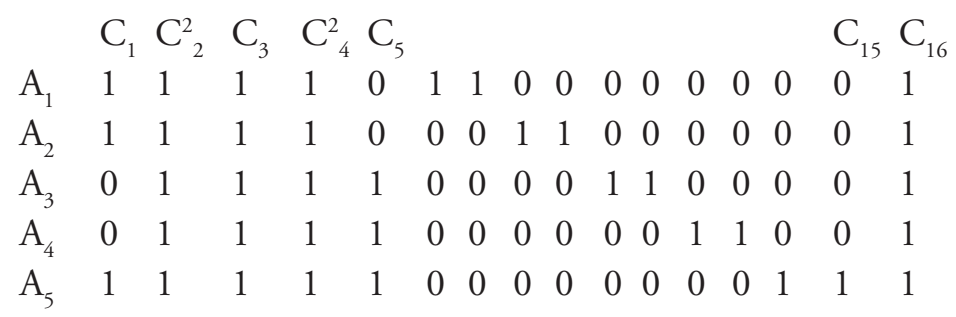

À cette date, l'anomalie $C_{16}$ est connue de tous, mais elle ne semble pouvoir être intégrée par aucun acteur dans la partie sémantiquement pertinente de son répertoire cognitif : pour $A_{1}$, elle serait, par exemple, sémantiquement cohérente avec $C_{7}$ mais non avec $C_{6}$; pour $A_{2}$, elle serait sémantiquement cohérente avec $C_{9}$ mais non avec $C_{8}$, et ainsi de suite jusqu'à $A_{5}$, pour lequel elle serait cohérente avec $C_{15}$ mais non avec $C_{14}$. Le fait que cette catégorie anormale soit désormais connue de tous milite en faveur de son intégration au socle de croyances partagées $p \mathrm{~S}_{\mathrm{C}}$

$78 \quad$ Ainsi, en 1887 l'expérience de Michelson-Morley a montré, à la stupéfaction des physiciens, que la vitesse de la lumière était la même dans toutes les directions, ce qui était contradictoire avec la théorie d'un éther supposé immobile et constituant un point fixe par rapport auquel on pouvait mesurer la vitesse de la Terre. Selon cette théorie en effet, si l'on mesurait la vitesse de la lumière transmise par l'éther, on devrait obtenir des mesures différentes selon que l'on va de la source lumineuse, avec la vitesse de la Terre autour du Soleil, ou qu'on la mesure dans une direction perpendiculaire à la tangente de l'orbite céleste. Or, l'expérience de Michelson-Morley montre que tel n'est pas le cas. Cette expérience fut réalisée un très grand nombre de fois dans des conditions très diverses, d'abord par Michelson-Morley (sous terre, sur une colline), puis par bien d'autres (au mont Wilson, à 1800 mètres d'altitude en 1921 et 1925; dans un ballon libre à 2500 mètres d'altitude par Picard en 1926-1928), et ceci jusqu'en 1958! Entre-temps, le concept d'éther était devenu très embarrassant, car les physiciens ne parvenaient à le conserver qu'en dépouillant l'éther de toutes ses propriétés mécaniques. La question de l'existence même de l'éther se posa alors, et Einstein la résolut par la négative en 1905 (Jacques Roger, "Le problème philosophique ", dans Émile Noël (dir.), L'espace et le temps aujourd'hui, Paris, Seuil, 1983, p. 39-49). 
$(\mathrm{t}+\mathrm{T})$, mais, d'un autre côté, elle n'est sémantiquement cohérente avec aucun $p \mathrm{~S}_{\mathrm{i}}(\mathrm{t}+\mathrm{T})$. Pour le paradigme en place, il s'ensuit une crise que nous pouvons formaliser par une scission de $p \mathrm{~S}_{\mathrm{C}}(t+T)$ en deux sous-ensembles parfaitement disjoints : $\left\{\mathrm{C}_{2}^{2}, \mathrm{C}_{3}, \mathrm{C}_{4}^{2}\right.$, $\left.\mathrm{C}_{2}^{2} \mathrm{C}_{3}, \mathrm{C}_{2}^{2} \mathrm{C}_{4}^{2}\right\}$ d'une part, et $\left\{\mathrm{C}_{16}\right\}$, d'autre part. Par ailleurs, les diverses interprétations du paradigme n'ont pas changé par rapport à celles qui prévalaient déjà à la date $t$, car elles sont toujours incapables d'intégrer l'anomalie $C_{16}$ :

$$
\begin{gathered}
p \mathrm{~S}_{1}(\mathrm{t}+\mathrm{T})=p \mathrm{~S}_{1}(\mathrm{t})=\left\{\mathrm{C}_{6}, \mathrm{C}_{2}^{2} \mathrm{C}_{6}, \mathrm{C}_{3} \mathrm{C}_{6}, \mathrm{C}_{2}{ }^{2} \mathrm{C}_{3} \mathrm{C}_{6}\right\} \\
p \mathrm{~S}_{2}(\mathrm{t}+\mathrm{T})=p \mathrm{~S}_{2}(\mathrm{t})=\left\{\mathrm{C}_{8}, \mathrm{C}_{2}{ }^{2} \mathrm{C}_{8}, \mathrm{C}_{3} \mathrm{C}_{8}, \mathrm{C}_{2}{ }^{2} \mathrm{C}_{3} \mathrm{C}_{8}\right\} \\
p \mathrm{~S}_{3}(\mathrm{t}+\mathrm{T})=p \mathrm{~S}_{3}(\mathrm{t})=\left\{\mathrm{C}_{10}, \mathrm{C}_{2}{ }^{2} \mathrm{C}_{10}, \mathrm{C}_{3} \mathrm{C}_{10}, \mathrm{C}_{2}{ }^{2} \mathrm{C}_{3} \mathrm{C}_{10}\right\} \\
p \mathrm{~S}_{4}(\mathrm{t}+\mathrm{T})=p \mathrm{~S}_{4}(\mathrm{t})=\left\{\mathrm{C}_{12}, \mathrm{C}_{2}{ }^{2} \mathrm{C}_{12}, \mathrm{C}_{3} \mathrm{C}_{12}, \mathrm{C}_{2}{ }^{2} \mathrm{C}_{3} \mathrm{C}_{12}\right\} \\
p \mathrm{~S}_{5}(\mathrm{t}+\mathrm{T})=p \mathrm{~S}_{5}(\mathrm{t})=\left\{\mathrm{C}_{14}, \mathrm{C}_{2}{ }^{2} \mathrm{C}_{14}, \mathrm{C}_{3} \mathrm{C}_{14}, \mathrm{C}_{2}{ }^{2} \mathrm{C}_{3} \mathrm{C}_{14}\right\}
\end{gathered}
$$

Cet état de crise incite les acteurs à s'interroger sur les fondements épistémologiques de leurs croyances, donc à expliciter et éventuellement à remettre en question les métacatégories qui gouvernent le mode de sélection et d'organisation des combinaisons de catégories élémentaires sémantiquement pertinentes composant ces croyances. Ramenées dans la mesure du possible par leur explicitation à un niveau conscient, ces métacatégories peuvent alors faire l'objet d'un travail de déconstruction. Certaines de celles dont le bien-fondé n'avait pas été suffisamment interrogé lors de la série de communications sociales qui les avait progressivement renforcées jusqu’à les faire accéder au métaniveau qui est le leur se voient reconsidérées et parfois rejetées. En termes de notre exemple, il se peut ainsi que l'on s'aperçoive qu'aucun singleton ne peut avoir de pertinence sémantique, contrairement à ce que l'on avait trop rapidement accepté jusque là. Pour l'ensemble des acteurs, le fait même que l'anomalie $C_{16}$ n'acquière une éventuelle pertinence sémantique qu'en étant combinée à une autre, même si celle-ci est différente selon les acteurs, est en effet de nature à jeter un doute sérieux sur la pertinence sémantique de toute catégorie élémentaire isolée. Une nouvelle norme fait ainsi son apparition en matière de sélection et d'organisation des catégories élémentaires dans les croyance des acteurs, qui 
stipule, par exemple, que toute partie sémantiquement pertinente des répertoires cognitifs doit combiner au moins deux catégories élémentaires puisées dans ces répertoires. Une telle modification implique une transformation radicale du paradigme précédent, construit sur la base d'une norme moins contraignante.

Dans notre exemple, cette transformation radicale se traduit par : i) la disparition de tout singleton des croyances des acteurs, mais non de leurs répertoires cognitifs; ii) la substitution, dans les nouvelles croyances ainsi formées des acteurs $A_{1}, A_{2}, A_{3}, A_{4}$ et $A_{5}$, des catégories respectives $C_{7}, C_{9}, C_{11}, C_{13}$ et $C_{15}$ aux catégories $C_{6}, C_{8}, C_{10}, C_{12}$ et $C_{14}$ qui figuraient à un titre autre que celui de singleton dans les croyances antérieures; iii) l'introduction de la catégorie $C_{16}$ dans certaines des combinaisons ainsi obtenues. Les répertoires cognitifs étant restés identiques à ceux correspondant à l'état du réseau à la date $t+T$, le fondement cognitif du nouveau paradigme $\mathscr{C}_{2}$ est alors représentable sous la forme suivante :

$$
\begin{aligned}
& \mathrm{pS}_{\mathrm{C}}(\mathrm{t}+\mathrm{T})=\left\{\mathrm{C}_{2}^{2} \mathrm{C}_{3}, \mathrm{C}_{2}^{2} \mathrm{C}_{4}^{2}, \mathrm{C}_{2}^{2} \mathrm{C}_{3} \mathrm{C}_{4}{ }_{4} \mathrm{C}_{16}\right\} \\
& \text { et } \\
& \mathrm{p} \mathrm{S}_{1}(\mathrm{t}+\mathrm{T})=\left\{\mathrm{C}_{2}^{2} \mathrm{C}_{7}, \mathrm{C}_{3} \mathrm{C}_{7}, \mathrm{C}_{7} \mathrm{C}_{16}, \mathrm{C}_{2}^{2} \mathrm{C}_{3} \mathrm{C}_{7} \mathrm{C}_{16}\right\} \\
& \mathrm{p} \mathrm{S}_{2}(\mathrm{t}+\mathrm{T})=\left\{\mathrm{C}_{2}^{2} \mathrm{C}_{9}, \mathrm{C}_{3} \mathrm{C}_{9}, \mathrm{C}_{9} \mathrm{C}_{16}, \mathrm{C}_{2}^{2} \mathrm{C}_{3} \mathrm{C}_{9} \mathrm{C}_{16}\right\} \\
& \mathrm{p} \mathrm{S}_{3}(\mathrm{t}+\mathrm{T})=\left\{\mathrm{C}_{2}^{2} \mathrm{C}_{11}, \mathrm{C}_{3} \mathrm{C}_{11}, \mathrm{C}_{11} \mathrm{C}_{16}, \mathrm{C}^{2}{ }_{2} \mathrm{C}_{3} \mathrm{C}_{11} \mathrm{C}_{16}\right\} \\
& \mathrm{pS}_{4}(\mathrm{t}+\mathrm{T})=\left\{\mathrm{C}_{2}^{2} \mathrm{C}_{13}, \mathrm{C}_{3} \mathrm{C}_{13}, \mathrm{C}_{13} \mathrm{C}_{16}, \mathrm{C}_{2}^{2} \mathrm{C}_{3} \mathrm{C}_{13} \mathrm{C}_{16}\right\} \\
& \mathrm{p} \mathrm{S}_{5}(\mathrm{t}+\mathrm{T})=\left\{\mathrm{C}_{2}^{2} \mathrm{C}_{15}, \mathrm{C}_{3} \mathrm{C}_{15}, \mathrm{C}_{15} \mathrm{C}_{16}, \mathrm{C}_{2}^{2} \mathrm{C}_{3} \mathrm{C}_{15} \mathrm{C}_{16}\right\}
\end{aligned}
$$

Ce nouvel ensemble de parties sémantiquement pertinentes est incommensurable avec l'ancien. Certes, il subsiste certaines combinaisons de catégories élémentaires communes à $\mathscr{C}_{1}$ et $\mathscr{C}_{2}$, telles $C_{2}^{2} C_{3}$ et $C_{2}^{2} C_{2}^{2}$, mais la possibilité de traduction est au mieux partielle entre les deux paradigmes successifs, car $C_{2}^{2} C_{3}$ et $C^{2}{ }_{2} C_{4}^{2}$ acquièrent une tout autre signification dans $C_{2}$ où elles apparaissent liées à d'autres comme dans $C_{2}^{2} C_{3} C_{4}^{2} C_{16}$ figurant dans $p \mathrm{~S}_{\mathrm{C}}(t+T)$, que dans $\mathscr{C}_{1}$ où elles apparaissaient séparées l'une de l'autre dans $p \mathrm{~S}_{\mathrm{C}}(t)$ ou, pour $C_{2}^{2} C_{3}$, liée à d'autres comme dans $C^{2}{ }_{2} C_{3} C_{6}$ figurant dans $p S_{1}(t), C^{2}{ }_{2} C_{3} C_{8}$ figurant dans $p \mathrm{~S}_{2}(t)$, etc. 
La logique de cet exemple est globalement conforme à celle d'une révolution scientifique au sens de Kuhn, et l'incommensurabilité des paradigmes successifs ici décrite contient les sept éléments principaux que les derniers textes de ce même auteur intégraient, selon Soler, dans la notion d'incommensurabilité. En particulier, le déclenchement de la crise au sein d'un paradigme existant est dû à l'apparition d'une anomalie qui a pris, dans notre exemple, la forme de l'apparition d'une nouvelle catégorie élémentaire $C_{16}$, d'abord idiosyncrasique, puis partagée par tous les acteurs. Dans la perspective plus large qui est la nôtre ici celle d'un apprentissage batesonien de niveau 3 pouvant s'incarner en une révolution scientifique à la Kuhn, mais aussi en une transformation radicale du mode de penser d'acteurs non scientifiques - il existe au moins une autre logique possible aboutissant au même type de résultats : celle de l'accession d'une (ou de plusieurs) catégorie(s) élémentaire(s) $C_{j}{ }^{g-1}$ au métaniveau non conscient suite à une $g$-ième occurrence de cette (ces) catégorie(s) dans un (ou plusieurs) répertoire(s) cognitif(s) individuel(s). Un tel passage du niveau catégoriel conscient au métaniveau catégoriel non conscient implique en effet une modification de la composition de l'ensemble des métacatégories de type $C_{j}^{g}$ sous forme d'adjonction d'un (ou de plusieurs) éléments nouveaux. Sauf à attribuer aux règles gouvernant ce niveau métacatégoriel une logique purement additive que nous avons refusée depuis le début de ce texte à celles qui gouvernent le niveau catégoriel, une telle modification ne peut pas ne pas déboucher sur un changement dans le mode de sélection et d'organisation des parties sémantiquement pertinentes des répertoires cognitifs individuels - sur une transformation radicale des supports cognitifs des paradigmes, conventions et consensus correspondants. En réalité, ce type de processus aboutit au même genre de résultats que ceux décrits par notre exemple au terme d'un cheminement exactement inverse : au lieu de consister en une explicitation de certaines métacatégories ainsi ramenées au niveau catégoriel conscient, il prend la forme d'une "implicitation " de certaines catégories ainsi hissées au niveau métacatégoriel non conscient. 
La conclusion générale que nous pouvons en tirer est alors la suivante : la condition nécessaire de la transformation radicale d'un paradigme scientifique ou d'un consensus public consiste en une modification (par ajout ou retrait d'au moins un élément) de l'ensemble des métacatégories gouvernant le mode de sélection et d'organisation des parties sémantiquement pertinentes des répertoires cognitifs individuels sous-jacents à ces types de collectifs. Ce sont alors la bande de Moebius liant le temps subjectif des acteurs observés au temps objectif de l'observateur du réseau, ainsi que la bouteille de Klein figurant le type de spatialisation du temps qui se noue lors de l'intersubjectivité entre observés et observateur, telle que l'observe ce troisième homme que nous sommes, qui se trouvent elles-mêmes changées. Car, contrairement aux processus de recombinaison et de renforcement de l'ancrage de catégories existantes, de tels changements sont totalement imprévisibles : aussi compliquées que soient les combinatoires des acteurs en communication et des contenus des messages échangés à cette occasion, les apprentissages de niveaux 1 et 2 résultant de la communication sociale se situent dans un espace de risque probabilisable, alors que l'apprentissage de niveau 3 relève de l'incertitude radicale, telle que Knight et Keynes en ont simultanément introduit le concept.

\section{Bibliographie}

Adde, Alain, Sur la nature du temps, Paris, PUF, 1998.

Alchourrón, Carlos, Peter Gärdenfors et David Makinson, « On the logic of theory change: Partial meet contraction and revision ", Journal of Symbolic Logic, vol. 50, 1985, p. 510-530.

Amin, Ash et Patrick Cohendet, Architectures of Knowledge, New York, Oxford University Press, 2004.

Ancori, Bernard, "Analogie, évolution scientifique et réseaux complexes », Nouvelles perspectives en sciences sociales. Revue internationale d'analyse complexe et d'études relationnelles, vol. 1, $\mathrm{n}^{\circ}$ 1, 2005, p. 9-62. 
Ancori, Bernard, "Analogie, métaphore et mutation féodale (XI ${ }^{\mathrm{e}}-\mathrm{XIII}{ }^{\mathrm{e}}$ siècle) ", Colloque Pour ou contre la métaphore? Pouvoir, histoire, savoir et poétique, Collège militaire royal du Canada, Kingston (Ontario), 22-25 mai, 2007, à paraître dans François-Emmanuel Boucher, Janusz Przychodzen et Sylvain David (dir.), Pour ou contre la métaphore?, Kingston (Ontario), octobre 2008).

Ancori, Bernard, "Apprentissage auto-organisationnel et problématique du choix individuel ", dans Bernard Ancori (dir.), Apprendre, se souvenir, décider. Une nouvelle rationalité de l'organisation, Paris, CNRS Éditions, 1992, p. 137-173.

Ancori, Bernard, Échange monétaire et évolution économique, Université Louis Pasteur (Strasbourg I), 1990.

Ancori, Bernard, "Évolution, complexité et consensus monétaire : un modèle théorique et quelques illustrations historiques ", Économie Appliquée, Tome I, n 3, 1997, p. 199-236.

Ancori, Bernard, "Mémoire et apprentissage : de la neurobiologie à l'autoorganisation ", dans Bernard Ancori (dir.), Apprendre, se souvenir, décider. Une nouvelle rationalité de l'organisation, Paris, CNRS Éditions, 1992, p. 51-104.

Ancori, Bernard, "Réseau complexe et genèse des conventions ", CDRom des Actes du $\sigma^{e}$ Congrès européen de science des systèmes, Paris, septembre 2005, p. 19-22.

Ancori, Bernard, "Sur le caractère dialectique du couple conceptuel controverse/consensus : un modèle théorique et quelques exemples issus de l'histoire des sciences et des Science Studies ", Journée d'étude "Matière à controverses ", Université Marc Bloch (Strasbourg II), 10 novembre, 2006 (à paraître sous le titre " Les dialectiques du couple conceptuel controverse/consensus. Un modèle théorique et quelques exemples (histoire des sciences, Science Studies ", dans Patrick Schmoll (dir.), Matières à controverses, Strasbourg, Presses de l'Université Marc Bloch (Strasbourg II), juin 2008).

Ancori, Bernard, Antoine Bureth et Patrick Cohendet, "The Economics of Knowledge: The Debate about Codification and Tacit Knowledge ", Industrial and Corporate Change, vol. 9, $\mathrm{n}^{\circ}$ 2, 2000, p. 255-287.

Arrow, Kenneth J., "Economic Welfare and the Allocation of Resources for Innovation ", dans Universities National Bureau Committee for Economic Research, The Rate and Direction of Inventive Activity, Princeton (New Jersey), Princeton University Press, 1962, p. 609625.

Atlan, Henri, «Les échanges de savoir : la bibliothèque comme métaphore d'organisation biologique ", Le genre humain, $n^{\circ} 33$ Interdisciplinarités 
hiver 1998, p. 197-204.

Atlan, Henri, "L'intuition du complexe et ses théorisations ", dans Françoise Fogelman Soulié (dir.), Les théories de la complexité. Autour de l'ouvre d'Henri Atlan, Paris, Seuil, 1991, p. 9-42.

Atlan, Henri, Entre le cristal et la fumée. Essai sur l'organisation du vivant, Paris, Seuil, 1979.

Atlan, Henri, L'organisation biologique et la théorie de l'information, Paris, Hermann, 1972.

Balian, Roger, "Le temps macroscopique ", dans Étienne Klein et Michel Spiro (dir.), Le temps et sa flèche, Paris, Flammarion, [1994] 1996, p. 155-211.

Barberousse, Anouk, Max Kistler et Pascal Ludwig, La philosophie des sciences au XXe siècle, Paris, Flammarion, 2000.

Bateson, Gregory, Une unité sacrée. Quelques pas de plus vers une écologie de l'esprit, Paris, Seuil, [éd. américaine : 1991] 1996.

Bateson, Gregory, La nature et la pensée, Paris, Seuil, [éd. américaine : 1979] 1984.

Bateson, Gregory, Vers une écologie de l'esprit, Tome II, Paris, Seuil, [éd. américaine : 1972] 1980.

Bateson, Gregory, Vers une écologie de l'esprit, Tome I, Paris, Seuil, [éd. américaine : 1972] 1977.

Bergson, Henri, "Le possible et le réel ", dans Henri Bergson (dir.), La pensée et le mouvant : essais et conférences, Paris, PUF, [1938] 1998.

Bergson, Henri, Essai sur les données immédiates de la conscience, Paris, PUF, [1938] 1993.

Bergson, Henri, Matière et mémoire, Paris, PUF, [1896] 1990.

Berthelot, Jean-Michel, "Épistémologie et sociologie de la connaissance scientifique ", Cahiers internationaux de sociologie, vol. CIX, juilletdécembre, 2000, p. 221-234.

Boschma, Ron, "Proximity and innovation: A critical assessment ", Regional Studies, vol. 39, n 1, 2005, p. 61-74.

Boschma, Ron, " Proximité et innovation ", Économie rurale, n 280, marsavril 2004, p. 8-24.

Bourdieu, Pierre, Ce que parler veut dire. L'économie des échanges linguistiques, Paris, Fayard, 1982.

Bourgine, Paul et Jean-Pierre Nadal, Cognitive Economics. An Interdisciplinary Approach, Berlin, Springer-Verlag, 2004.

Boyer, Robert et André Orléan, "Persistance et changement des conventions. Deux modèles simples et quelques illustrations ", dans André 
Orléan (dir.), Analyse économique des conventions, Paris, PUF, 1994, p. 219-247.

Brillouin, Léon, La science et la théorie de l'information, Paris, Masson, 1959.

Bruner, Jérome S., "Les processus de préparation à la perception ", dans François Bresson, Jérome S. Bruner, Albert Morf et Jean Piaget (dir.), Logique et perception, Paris, PUF, 1958.

Callon, Michel et Bruno Latour, La science telle qu'elle se fait. Anthologie de la sociologie des sciences de langue anglaise, Paris, La Découverte, 1991.

Capra, Fritjof, Les connexions invisibles. Une approche systémique du développement durable, Monaco, Éditions du Rocher, [éd. américaine : 2002] 2004.

Capra, Fritjof, La Toile de la vie. Une nouvelle interprétation scientifique des systèmes vivants, Monaco, Éditions du Rocher, [éd. américaine : 1996] 2003.

Carnap, Rudolf, Logical Foundations of Probability, Chicago, University of Chicago Press, 1950.

Castoriadis, Cornelius, Le monde morcelé. Les carrefours du labyrinthe III, Paris, Seuil, 1990.

Chevalley, Catherine, Entrée, «Épistémologie », dans Barbara Cassin (dir.), Vocabulaire européen des philosophies. Dictionnaire des intraduisibles, Paris, Seuil/Le Robert, 2004, p. 358-366.

Colonna, Lucien, "Le temps psychologique ", dans Adolphe Pacault et Christian Vidal (dir.), À chacun son temps, Paris, Flammarion, 1975, p. 214-234.

Dasgupta, Partha et Paul A. David, "Toward a new economics of science ", Research Policy, vol. 23, n 5, 1994, p. 487-521.

Debreu, Gérard, Théorie de la valeur. Analyse axiomatique de l'équilibre général, Paris, Dunod, 1966.

Debru, Claude, «La conscience du temps. De la phénoménologie à la cognition ", dans "Neurosciences et Philosophie. Le problème de la conscience ", Revue de métaphysique et de morale, avril-juin, 1992, p. 273-93.

Degenne, Alain et Michel Forsé, "Comment on trouve ses amis. Enquête sur la sociabilité des Français ", Les liens sociaux invisibles, numéro hors série de Sciences humaines $\mathrm{n}^{\circ}$ 5, mai-juin 1994, p. 20-24.

Degenne, Alain et Michel Forsé, Les réseaux sociaux, Paris, Armand Colin, 1994.

Delahaye, Jean-Paul, "La ressemblance mathématisée », dans Jean-Paul 
Delahaye (dir.), L'intelligence et le calcul, de Gödel aux ordinateurs quantiques, Paris, Pour la Science, 2002, p. 21-27.

Delahaye, Jean-Paul, Information, complexité et hasard, London, Hermès Sciences Publishing Ltd, 1999.

Dubucs, Jacques, "La connaissance interactive ", Les chemins de la logique, Dossier Pour la Science, vol. 49, octobre-décembre 2005, p. 80-86.

Dupuy, Jean-Pierre, "Vers l'unité des sciences sociales autour de l'individualisme méthodologique complexe ", Revue du M. A. U. S. S., Une théorie sociologique générale est-elle pensable? De la science sociale ${ }^{\circ} 24$, second semestre, 2004, p. 310-328.

Edelman, Gérald M., Biologie de la conscience, Paris, Odile Jacob, [éd. anglaise : 1992] 1992.

Elias, Norbert, Du temps, Paris, Fayard, [éd. allemande : 1984] 1996.

Feyerabend, Paul K., "Explication, réduction et empirisme », dans Paul K. Feyerabend (dir.), Réalisme, rationalisme et méthode scientifique. Écrits philosophiques I, Chennevières-sur-Marne (Val-de-Marne), Éditions Dianoïa, [1962] 2005, p. 87-146.

Fleck, Ludwik, Genèse et développement d'un fait scientifique, Les Belles Lettres, [1935] 2005.

Fogelman Soulié, Françoise (dir.), Les théories de la complexité. Autour de l'auvre d'Henri Atlan, Paris, Seuil, 1991.

Foray, Dominique, L'économie de la connaissance, Paris, La Découverte, 2000.

Gaudin, Thierry, Introduction à l'économie cognitive, Nîmes, Éditions de l'Éclat, 1997.

Gell-Mann, Murray, Le quark et le jaguar. Voyage au cour du simple et du complexe, Paris, Albin Michel, [éd. américaine : 1994] 1995.

Georgescu-Roegen, Nicholas, The Entropy Law and the Economic Process, Cambridge (Massachusetts), Harvard University Press, 1971.

Georgescu-Roegen, Nicholas, La science économique. Ses problèmes et ses difficultés, Paris, Dunod, [1966] 1969.

Gil, Fernando, Article, "Catégories ", Encyclopedia Universalis, Tome IV, p. 88-91.

Goffman, Erving, Les cadres de l'expérience, Paris, Minuit, [1974] 1991.

Goldmann, Lucien, "L'importance du concept de conscience possible pour la communication ", Le concept d'information dans la science contemporaine, Cahiers de Royaumont $n^{\circ} \mathrm{V}$, Paris, Minuit, 1965, p. 47-77.

Goux, Jean-Joseph, "Cash, check or charge? ", dans Martin Gorin (dir.), L'argent, Communications, ${ }^{\circ}$ 50, Paris, Seuil, 1989, p. 7-22. 
Granovetter, Marc, "Les institutions économiques comme constructions sociales; un cadre d'analyse ", dans André Orléan (dir.), Analyse économique des conventions, Paris, PUF, 1994, p. 79-94.

Israël, Giorgio, La mathématisation du réel. Essai sur la modélisation mathématique, Paris, Seuil, 1996.

Jurdant, Baudouin, "La monnaie est-elle un langage? ", Fundamenta Sciente, vol. 9, nos 2-3, 1988, p. 285-296.

Jurdant, Baudouin, "The Role of Vowels in Alphabetic Writing ", dans Derrick de Kerckhove et Charles J. Lumsden (dir.), The Alphabet and the Brain: The Lateralization of Writing, Berlin, Springer-Verlag, 1988, p. 381-400.

Jurdant, Baudouin, "Écriture, monnaie et connaissance ", Thèse de Doctorat ès lettres, Université Louis Pasteur (Strasbourg I), 1984.

Katsuno, Hiroshi et Alberto Mendelzon, "On the difference between updating a knowledge base and revisiting it ", dans Peter Gärdenfors (dir.), Belief Revision, Cambridge, Cambridge University Press, 1992, p. 183-203.

Keynes, John Maynard, "A Treatise on probability ", The Collected Writtings of. J. M. Keynes, vol. 8, Macmillan London for the Royal Economic Society, [éd. anglaise : 1921] 1973.

Klir, George Jiri, "Les multiples visages de la complexité ", dans Edgar Morin (dir.), Science et pratique de la complexité, Actes du Colloque de Montpellier, mai 1984, Paris, La Documentation Française, 1986, p. 101-120.

Knight, Frank H., Risk, uncertainty and profit, Boston, Houghton Mifflin Company, 1921.

Kuhn, Thomas S., La structure des révolutions scientifiques, Paris, Flammarion, [éd. américaine : 1962] 1972.

Latour, Bruno, Changer de société. Refaire de la sociologie, Paris, La Découverte, [éd. anglaise : 2005] 2006.

Laugier, Sandra et Pierre Wagner (dir.), Philosophie des sciences. Volume I, Théories, expériences et méthodes, Paris, Vrin, 2004.

Laugier, Sandra et Pierre Wagner (dir.), Philosophie des sciences. Volume II, Naturalismes et réalismes, Paris, Vrin, 2004.

Le Moigne, Jean-Louis, La théorie du système général. Théorie de la modélisation, Paris, PUF, [1977] 1994.

Le Ny, Jean-François, Comment l'esprit produit du sens. Notions et résultats des sciences cognitives, Paris, Odile Jacob, 2005.

Lecourt, Dominique (dir.), Dictionnaire d'histoire et philosophie des sciences, Paris, PUF, 1999. 
Lévy-Leblond, Jean-Marc, "Quel temps fait-on? », dans Étienne Klein et Michel Spiro (dir.), Le temps et sa flèche, Paris, Flammarion, [1994] 1996, p. 271-81.

Lévy-Leblond, Jean-Marc, "La structure de l'espace-temps (les relativités) », dans Émile Noël (dir.), L'espace et le temps aujourd'hui, Paris, Seuil, 1983, p. 51-64.

Li, Ming et Paul Vitányi, An Introduction to Kolmogorov complexity and its applications, [Second Edition] Berlin, Springer-Verlag, 1997.

Lloyd, Geoffrey Ernest Richard, Pour en finir avec les mentalités, Paris, La Découverte, 1993.

Lloyd, Geoffrey Ernest Richard, Magie, raison et expérience. Origines et développement de la science grecque, Paris, Flammarion, 1990.

Lloyd, Geoffrey Ernest Richard, Les débuts de la science grecque de Thalès à Aristote, Paris, Maspéro, 1974.

Maffesoli, Michel, La transfiguration du politique. La tribalisation du monde, Paris, Grasset \& Fasquelle, 1992.

Maffesoli, Michel, Le temps des tribus. Le déclin de l'individualisme dans les sociétés de masse, Paris, Librairie des Méridiens, Klincksieck et Cie, 1988.

Morin, Edgar, Introduction à la pensée complexe, Paris, ESF, 1990.

Moulines, Carlos Ulises, La philosophie des sciences. L'invention d'une discipline (fin XIX'e-début XXIe siècle), Paris, Éditions Rue d'Ulm/Presses de l'École normale supérieure, 2006.

Nelson, Richard, "The Simple Economics of Basic Scientific Research ", Journal of Political Economy, vol. 67, 1959, p. 297-306.

Nowotny, Helga, Le temps à soi. Genèse et structuration d'un sentiment du temps, Paris, Éditions de la Maison des sciences de l'Homme, [1989] 1992.

Overbeke, Maurice van, "Temps et tension. À propos de deux relativités ", dans Enrico Castelli (dir.), Temporalité et aliénation, Paris, Aubier, 1975, p. 73-92.

Pestre, Dominique, Introduction aux Social Studies, Paris, La Découverte, 2006.

Piettre, Bernard, Philosophie et science du temps, Paris, PUF, 1994.

Polanyi, Michael, The Tacit Dimension, Oxford, Routledge \& Kegan, 1966.

Polanyi, Michael, Personal Knowledge, Oxford, Routledge, 1962.

Poplin, François, «Le parapluie et le bédane. Un lien anthropozoologique entre les canards et les chevaux ", Alliage nos 29-30, 1996-1997, p. 29-30. 
Popper, Karl, Les deux problèmes fondamentaux de la théorie de la connaissance, [Texte établi par Troels Eggers Hansen à partir de manuscrits des années 1930-1933] Paris, Hermann, 1999.

Popper, Karl, Toute vie est résolution de problèmes. Questions autour de la connaissance de la nature, Arles, Actes Sud, [éd. allemande : 1994] 1997.

Popper, Karl, Un univers de propensions. Deux études sur la causalité et l'évolution, Nîmes, Éditions de l'Éclat, 1992.

Popper, Karl, Post-scriptum à la logique de Paris. La Découverte scientifique. I, Le réalisme et la science, Paris, Hermann, [éd. anglaise : 1983] 1990.

Popper, Karl, Conjectures et réfutations. La croissance du savoir scientifique, Paris, Payot, [éd. anglaise : 1963] 1985.

Popper, Karl, La logique de Paris. La Découverte scientifique, Paris, Payot, [éd. allemande : 1934] 1973.

Popper, Karl, Objective Knowledge, Oxford, Clarendon Press, 1972.

Porge, Eric, Se compter trois. Le temps logique de Lacan, Ramonville, Éditions érès, 1989.

Prigogine, Israël et Isabelle Stengers, Entre le temps et l'éternité, Paris, Fayard, 1988.

Quine, Willard-V., "L'épistémologie naturalisée ", dans Sandra Laugier et Pierre Wagner (dir.), Philosophie des sciences, Volume II, Naturalismes et réalismes, Paris, Vrin, 2004, p. 36-60.

Quine, Willard-V., Quiddités. Dictionnaire philosophique par intermittence, Paris, Seuil, [éd. américaine : 1987] 1992.

Roger, Jacques, "Le problème philosophique ", dans Émile Noël (dir.), L'espace et le temps aujourd'hui, Paris, Seuil, 1983, p. 39-49.

Rosenfield, Israël, Une anatomie de la conscience. L'étrange, le familier, l'oublié, Paris, Flammarion, [éd. américaine : 1992] 1996.

Rosenfield, Israël, La Conscience. Une biologie du moi, Paris, Eshel, 1990.

Rosenfield, Israël, L'Invention de la mémoire. Le cerveau, nouvelles donnes, Paris, Eshel, [éd. américaine : 1988] 1989.

Ruyer, Raymond, La cybernétique et l'origine de l'information, Paris, Flammarion, 1954.

Sara, Susan, "Retrieval and Reconsolidation: Toward a Neurobiology of Remembering ", Learning \& Memory, vol. 7, n² 2, 2000, p. 73-84.

Schneider, Walter et Richard M. Shiffrin, " Controlled and automatic human information processing I: Detection, search, and attention", Psychological Review, vol. 84, 1977, p. 1-66. 
Schneider, Walter et Richard M. Shiffrin, "Controlled and automatic human information processing II: Perceptual learning, automatic attending, and a general theory ", Psychological Review, vol. 84, 1977, p. 127-90.

Shannon, Claude et Warren Weaver, Théorie mathématique de la communication, Paris, Retz, [éd. américaine : 1949] 1975.

Simon, Herbert, "The architecture of complexity ", Proceedings of the American Philosophy Society, vol. 106, 1962, p. 467-482. Reproduit dans Herbert Simon (dir.), Sciences des systèmes, Sciences de l'artificiel, Paris, Bordas, 1991, p. 171-203.

Soler, Léna, "Établir des correspondances entre théories scientifiques incommensurables? ", Revue philosophique de Louvain, vol. 102, n 3 , août 2004, p. 446-476.

Soler, Léna, "The Incommensurability Problem: Evolution, Current Approaches and Recent Issues ", dans Léna Soler (dir.), Le problème de l'incommensurabilité, un demi siècle après, Éditions Kimé, 2004, p. 1-38.

Soler, Léna, "De la commune mesure phénoménale des théories dites incommensurables ", Philosophia Scientic, vol. 7, 2003, p. 239-265.

Soler, Léna, Introduction à l'épistémologie, Paris, Ellipses, 2000.

Soler, Léna, "Le concept kuhnien d'incommensurabilité reconsidéré à la lumière d'une théorie structurale de la signification ", Philosophia Scientiae, vol. 4, n 2, 2000, p. 189-217.

Sperber, Dan (dir.), Metarepresentations: A Multidisciplinary Perspective, Oxford, Oxford University Press, 2000.

Sperber, Dan, "Individualisme méthodologique et cognitivisme ", dans Raymond Boudon, François Chazel et Alban Bouvier (dir.), Cognition et sciences sociales, Paris, PUF, 1997, p. 123-136.

Sperber, Dan, La contagion des idées. Théorie naturaliste de la culture, Paris, Odile Jacob, 1996.

Sperber, Dan et Deidre Wilson, La pertinence. Communication et cognition, Paris, Éditions de Minuit, [éd. anglaise : 1986] 1989.

Varela, Francisco, «Le présent spécieux : une neurophénoménologie de la conscience du temps ", dans Francisco Varela, Jean Petitot, Bernard Pachoud et Jean-Michel Roy (dirs.), Naturaliser la phénoménologie. Essais sur la phénoménologie contemporaine et les sciences cognitives, Paris, CNRS Éditions, 2002, p. 341-406.

Vinck, Dominique, Sciences et société. Sociologie du travail scientifique, Paris, Armand Colin, 2007.

Vinck, Dominique, Sociologie des sciences, Paris, Armand Colin, 1995. 
Walliser, Bernard, L'économie cognitive, Paris, Odile Jacob, 2000.

Walliser, Bernard et Paul Bourgine (dir.), Economics and Cognitive Science, Oxford, Pergamon Press, 1992.

Walliser, Bernard, Denis Zwirn et Hervé Zwirn, "Abductive logics in a belief revision framework ", Journal of Logic, Language and Information, vol. $14, \mathrm{n}^{\circ} 1,2005$, p. 87-117.

Weaver, Warren, "Science and complexity ", American Scientist, vol. 36, 1948, p. 536-544.

Winkin, Yves, Anthropologie de la communication. De la théorie au terrain, Bruxelles, De Boeck Université, 1996.

Winkin, Yves, La nouvelle communication, Textes recueillis et présentés par Yves Winkin, Paris, Seuil, 1981.

Zwirn, Denis et Hervé Zwirn, «La révision des croyances ", Pour la science, $n^{\circ} 311$, septembre 2003, p. 64-69. 